\title{
Mass transport and set-ups due to partial standing surface waves in a two-layer viscous system
}

\author{
By CHIU-ON NG \\ Department of Mechanical Engineering, The University of Hong Kong, Pokfulam Road, Hong Kong
}

(Received 24 November 2003 and in revised form 9 August 2004)

This is a theoretical study on the mass transport due to partially reflected long surface waves in a two-layer viscous system, which can be closed or open at its far end. Based on Lagrangian coordinates, a perturbation analysis is carried out to the second order to find the mean Lagrangian drifts in the two layers, where the lower fluid is taken to be much more viscous than the upper one. The free-surface and interfacial set-ups are also found as part of the solutions. A single analytical expression is obtained for the mass transport velocity in each layer, incorporating all the cases where the wave can be progressive, standing or partially standing, and the domain can be closed or open so that a return current may or may not exist. Through some numerical calculations, the patterns of flow in the recirculation cells due to the standing component of the wave, and in the unidirectional drifts due to the progressive component of the wave in a closed system are shown to vary with the lower-layer fluid viscosity. It is possible that, under some specific conditions, the mass transport in the core region of the upper layer is completely quiescent despite the existence of some strong drifts in the lower layer. The mean flow structures in the two layers can also respond rather differently to a change in the reflection coefficient in the presence or the absence of the return current.

\section{Introduction}

Mass transport in surface gravity waves is a long-standing and well-studied problem in ocean science and engineering. The core subject matter is how Lagrangian drifts are produced under various geophysical settings in the interior of a body of fluid due to small-amplitude periodic surface gravity waves. It was pointed out by LonguetHiggins (1953) that the mass transport velocity cannot be correctly predicted by the irrotational theory alone, and the viscous flow in boundary layers of the fluid must be taken into account. The wave boundary layers, also called Stokes boundary layers, are the primary places where second-order steady streaming and vorticity are generated. The transport of vorticity from the boundary layers into the fluid core is then carried out by viscous diffusion and convection, which are the respective dominant mechanisms when the wave amplitude is much smaller or greater than the Stokes boundary layer thickness. Longuet-Higgins (1953) hence called the solutions corresponding to these two cases the 'conduction' and 'convection' solutions.

Since the pioneering work of Longuet-Higgins (1953), a Lagrangian stream function has been used as a variable in the mathematical formulation for the problem of two-dimensional mass transport in waves. To uniquely determine the stream function, Longuet-Higgins and many others since then have made the assumption that the total 
horizontal flow due to the mass transport is zero. This amounts to assuming a zero value for the stream function at both the bottom and the free surface of the fluid layer. Re-examining the problem based on Lagrangian equations of motion, Ünlüata \& Mei (1970) later pointed out that this condition of zero net flux corresponds to a domain closed at the far end and is satisfied when a return current, induced by a mean horizontal pressure gradient, is established to balance the forward current. They therefore also obtained the mass transport velocity in an open system, which is not readily available using the stream function formulation alone, by simply putting the mean pressure gradient to zero. It was pointed out recently by $\mathrm{Ng}(2004 a)$ that it is not exactly the Lagrangian mean pressure gradient, but the mean displacement of the free surface, or the free surface set-up, that is responsible for the return current. It is only in the Eulerian system, as in Wen \& Liu (1995), that the return current can be regarded to be solely due to the mean pressure gradient.

With a view improving some deficiencies in the existing literature, $\mathrm{Ng}(2004 a)$ has revisited the classical problem of mass transport due to surface waves in a single-layer system. We here intend to extend his work to a two-layer viscous system. One of the objectives is to make clear the roles played by the free surface and interfacial set-ups in the mass transport problem, and to show the proper way of deriving these set-ups in the course of finding the mass transport velocity. Piedra-Cueva (1995) has presented a similar study on the mass transport for progressive waves in a two-layer system. He used a questionable method to find the interfacial set-up however. It has been shown by $\mathrm{Ng}(2004 a)$ that the Lagrangian expression for the set-up is not the same as the Eulerian counterpart. Piedra-Cueva (1995) has introduced an Eulerian equation for the Lagrangian set-up of the interface; this is inconsistent. In this work, partially reflected surface waves are considered, with the pure progressive and pure standing waves being the two limiting cases. We shall derive for each fluid a single expression for the mass transport velocity encompassing both possible (i.e. open and closed) far end conditions of the domain. To this end, the long-wave approximation is employed in this work to simplify the analysis. A stream function can be avoided, and expressions for the various set-ups are found more straightforwardly.

We also present a detailed numerical discussion on the mass transport in the two layers when subject to changes in environmental factors like the wave reflection, the lower-layer fluid viscosity (such that the one-layer system is a limiting case), and the far-end condition of the domain. We shall in particular look into how the viscosity of the lower-layer fluid will affect the sense of rotation of the recirculation cells when under a standing wave, and the direction of the horizontal drifts when under a progressive wave in a closed system. The transition of the mean Lagrangian flow field from recirculation cells to unidirectional drifts when the reflection coefficient varies between 1 and 0 is also examined for a domain that can be closed or open. In other existing theoretical developments on mass transport in a two-layer system, which include Dore (1970, 1973, 1976a, 1978), Crampin \& Dore (1979), and Wen \& Liu (1995), only either pure progressive or pure standing waves were considered. Most of them also only considered closed systems. Iskandarani \& Liu (1991) have studied mass transport due to partially reflected waves, but only for a closed single-layer system.

We shall follow Ünlüata \& Mei (1970) and Piedra-Cueva (1995) in employing the Lagrangian approach for the analysis. Also, like Piedra-Cueva (1995), we shall consider a layered system consisting of a very low-viscosity fluid (e.g. clear water) overlying a highly viscous fluid (e.g. concentrated fluid mud). The viscosity effect is reflected by the ratio of the Stokes boundary layer thickness to the respective fluid depth; the ratio is much smaller than unity for the upper fluid, but is of order unity for 
the lower fluid. Therefore, to the leading order, viscosity is important for the upper fluid only within oscillatory boundary layers near the interface and the free surface of the fluid, but is important across the entire layer of the lower fluid. The use of Lagrangian coordinates is to enable the imposition of the boundary conditions exactly on the free surface and the interface, rather than their equilibrium levels as in an Eulerian analysis, thereby eliminating the restriction that the wave amplitude must be smaller than the Stokes boundary layer thickness. This approach is alternative to the curvilinear coordinate system introduced by Longuet-Higgins (1953).

We disagree with Piedra-Cueva (1995) however in claiming that the convection of the second-order vorticity from a boundary layer into the interior region, which is appreciable when the wave amplitude is comparable to the Stokes boundary layer thickness, is taken fully into account implicitly in the solutions based on the direct use of Lagrangian coordinates. As given in Mei (1989), the solutions that are obtainable based on the Lagrangian perturbation expansions of Pierson (1962) are only for the creeping flow of the mass transport in the interior region. The solutions, which are hence the conduction solutions, apply when only viscous diffusion is taken into account in the transport of vorticity. In fact, the nonlinear effects of convection, or inertia more exactly, in an Eulerian formulation will show up in an even more intricate way through different terms in a Lagrangian formulation. If not strictly one-dimensional as in a progressive wave, the mass transport problem in either formulation will become nonlinear when the inertia becomes significant. To find the convection solution in the Eulerian system, past researchers have resorted to the double boundary-layer theory (Dore 1976a, b; Crampin \& Dore 1979) and numerical techniques like the spectral and finite-element methods (Iskandarani \& Liu 1991). The convection solution, which features a double structure of boundary layers, does not seem to have been sought in terms of Lagrangian coordinates in the literature.

To find the conduction solutions to the present problem, it is assumed that the surface wave amplitude is smaller than the Stokes boundary layer thickness in the lower viscous fluid. We do not consider that this condition will necessarily limit the present problem to extremely small waves. It is common to find in practice a fluid mud which is so highly viscous (Mei \& Liu 1987; Sakakiyama \& Bijker 1989) that the corresponding Stokes boundary layer thickness, for sufficiently long waves, can be as much as $O(1) \mathrm{m}$. Therefore under small-amplitude waves in shallow layers, it is reasonable to expect that the entire field of mean motions in a very viscous layer is dominated by viscous diffusion. Since the water viscosity is much lower than that of the lower fluid, it is possible however that inertia can have some effects on the mean motions in the water core region. Nevertheless, as illustrated by the numerical results of Iskandarani \& Liu (1991), such inertia effects are typically very limited (especially for a low reflection coefficient) if the wave amplitude is not too much larger than the Stokes boundary layer thickness. In this work, we shall suppose that the latter condition is true in the water layer, for which the inertia nonlinearity is thereby ignored for simplicity.

Our problem is further specified in the next section, where the long-wave approximation is applied to the Lagrangian form of the equations of motion and boundary conditions. Based on the assumption of small-amplitude displacements, a perturbation analysis is then carried out. Solutions to the first-order motions, which are purely oscillatory, are developed in $\S 3$. The second-order mean motions are then found in $\S 4$ for the upper and the lower layers, where the upper layer is further divided into the core region and the interfacial boundary layer. Particular attention is paid to the relationships between the various components of the free surface and interfacial set-ups and the inviscid and viscous parts of the flow induced by the waves. In the 

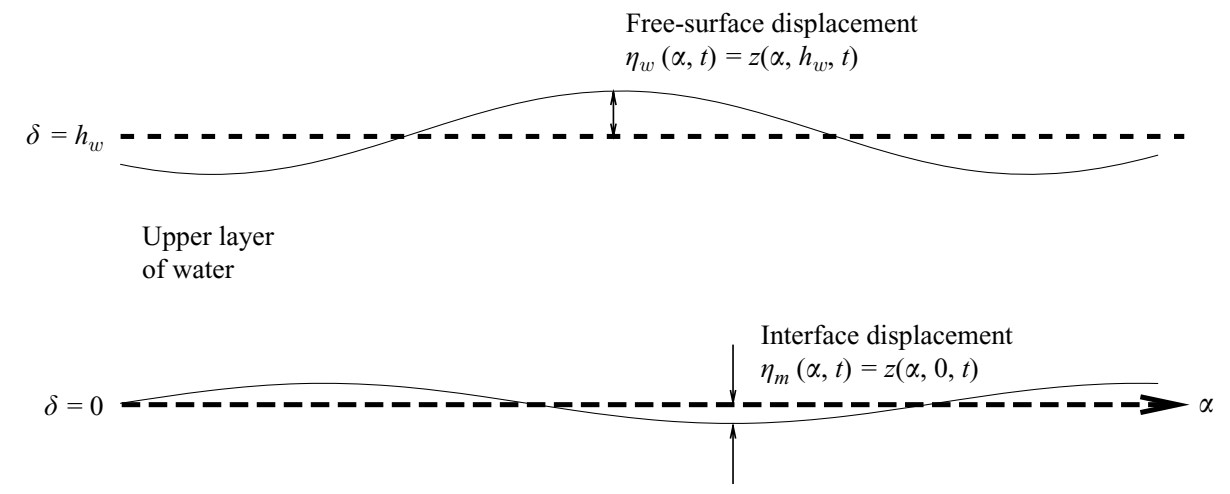

Lower layer of a very viscous fluid

particle displacements

$x=x(\alpha, \delta, t)$

$z=z(\alpha, \delta, t)$

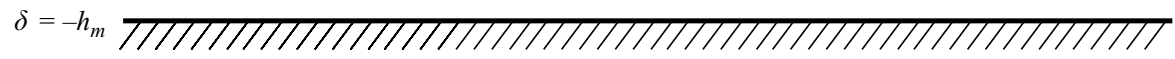

FIGURE 1. Sketch of a two-layer fluid system under surface waves.

end, a single expression is derived for the mass transport velocity in each layer that incorporates all the cases where the wave can be progressive, standing or partially standing and the system can be open or closed. Finally, in $\S 5$, numerical results are generated to enable a discussion on the effects due to the viscosity of the lower-layer fluid and the reflection coefficient on the mean Lagrangian flow field in the two-layer system in the presence or the absence of the return current.

\section{Mathematical formulation of the problem}

As sketched in figure 1, we consider a two-layer stratified system in which the upper layer is clear water and overlies a layer of highly viscous fluid, the depths of the two layers being $h_{w}$ and $h_{m}$, respectively. It is assumed that the fluids are homogeneous and separated by a sharp interface with little mixing. Although the bottom viscous fluid layer is typically thin, we allow for theoretical generality the two layer thicknesses to be comparable with each other: $h_{w} \approx h_{m}$. We refer the equations of motion to Lagrangian coordinates $(\alpha, \delta)$, which are the undisturbed horizontal/vertical positions of a fluid particle. The $\delta$-axis is directed vertically upwards with the origin $\delta=0$ fixed at the equilibrium level of the interface. The current position of a particle, denoted by $(x, z)$, is a function of $\alpha, \delta$ and time $t \geqslant 0$. Fluid particles are set in motion owing to a partial standing wave resulting from the superposition of two waves propagating in opposite directions on the free surface of the upper layer:

$$
\eta_{w}(\alpha, t)=\operatorname{Re}\left[a\left\{\mathrm{e}^{\mathrm{i}(k \alpha-\sigma t)}+R \mathrm{e}^{\mathrm{i}\left(k^{*} \alpha+\sigma t\right)}\right\}\right]
$$

where $a$ is the amplitude of the forward-going wave, i is the complex unit, $R$ is the reflection coefficient or the ratio of the wave amplitudes, $k$ is the wavenumber, and $\sigma$ is the angular frequency. An asterisk is used to denote the complex conjugate. While $a$ and $\sigma$ are some real and prescribed constants, $k$ is a complex eigenvalue to be determined by a dispersion relation, as will be deduced later. The reflection coefficient is in general complex, but its imaginary part can be removed by a proper choice of the origins for $\alpha$ and $t$ (Mei 1989). Therefore $R$ is here taken to be a prescribed real constant as well, and the wave is in general a partial standing wave with $0<R<1$. In 
the two limits, the wave becomes purely progressive when $R=0$, and purely standing when $R=1.0$.

As in any two-layer system, two modes of wave motions (surface and interfacial modes) are possible. Since our attention is limited to long waves, only the surface mode, in which the free-surface wave amplitude is larger than that of the interfacial wave, is of interest and significance here. For long waves of small amplitude, the ratio of the total thickness $h=h_{w}+h_{m}$ to the wavelength is a very small parameter

$$
\epsilon \equiv|k h| \ll 1,
$$

which will be used as the ordering parameter in the present analysis. Under the long-wave limit, some terms in the equations of motion and boundary conditions become subdominant. These equations in Lagrangian form, which are obtainable from Pierson (1962) and Piedra-Cueva (1995), can be written as follows, in which $\epsilon$ is inserted for identification of the order of the subdominant terms. Using the standard notation for a Jacobian, the mass conservation equation is

$$
\frac{\partial\left(x_{f}, z_{f}\right)}{\partial(\alpha, \delta)}=1
$$

where the subindex $f$ is used to distinguish between the upper and lower fluid domains

$$
f= \begin{cases}w & \text { for water in } h_{w}>\delta>0, \\ m & \text { for highly viscous fluid in } 0>\delta>-h_{m} .\end{cases}
$$

The $x$ - and $z$-momentum equations are

$$
\begin{gathered}
\ddot{x}_{f}=-\frac{1}{\rho_{f}} \frac{\partial\left(p_{f}, z_{f}\right)}{\partial(\alpha, \delta)}+\frac{1}{\rho_{f}}\left[\epsilon^{2} \frac{\partial\left(\tau_{f x x}, z_{f}\right)}{\partial(\alpha, \delta)}+\frac{\partial\left(x_{f}, \tau_{f x z}\right)}{\partial(\alpha, \delta)}\right], \\
\epsilon^{2} \ddot{z}_{f}=-\frac{1}{\rho_{f}} \frac{\partial\left(x_{f}, p_{f}\right)}{\partial(\alpha, \delta)}-g+\epsilon^{2} \frac{1}{\rho_{f}}\left[\frac{\partial\left(\tau_{f z x}, z_{f}\right)}{\partial(\alpha, \delta)}+\frac{\partial\left(x_{f}, \tau_{f z z}\right)}{\partial(\alpha, \delta)}\right],
\end{gathered}
$$

where the stress components are

$$
\begin{gathered}
\tau_{f x x}=2 \mu_{f} \frac{\partial\left(\dot{x}_{f}, z_{f}\right)}{\partial(\alpha, \delta)}, \\
\tau_{f z z}=2 \mu_{f} \frac{\partial\left(x_{f}, \dot{z}_{f}\right)}{\partial(\alpha, \delta)}, \\
\tau_{f x z}=\tau_{f z x}=\mu_{f}\left[\frac{\partial\left(x_{f}, \dot{x}_{f}\right)}{\partial(\alpha, \delta)}+\epsilon^{2} \frac{\partial\left(\dot{z}_{f}, z_{f}\right)}{\partial(\alpha, \delta)}\right] .
\end{gathered}
$$

In the equations above, an overdot is used to denote time derivative, $g$ is the acceleration due to gravity, $p$ is the pressure, $\rho$ is the fluid density, and $\mu$ is the dynamic viscosity. The boundary conditions can be written in a more compact form if we first give the following expressions for the stress components that are respectively tangential and normal to a material curve:

$$
\begin{gathered}
T_{f}=\epsilon^{2}\left(\tau_{f z z}-\tau_{f x x}\right) \frac{\partial x}{\partial \alpha} \frac{\partial z}{\partial \alpha}+\tau_{f x z}\left[\left(\frac{\partial x}{\partial \alpha}\right)^{2}-\epsilon^{2}\left(\frac{\partial z}{\partial \alpha}\right)^{2}\right] \\
N_{f}=-p_{f}\left[\left(\frac{\partial x}{\partial \alpha}\right)^{2}+\epsilon^{2}\left(\frac{\partial z}{\partial \alpha}\right)^{2}\right] \\
+\epsilon^{2}\left[\epsilon^{2} \tau_{f x x}\left(\frac{\partial z}{\partial \alpha}\right)^{2}+\tau_{f z z}\left(\frac{\partial x}{\partial \alpha}\right)^{2}-2 \tau_{f x z} \frac{\partial x}{\partial \alpha} \frac{\partial z}{\partial \alpha}\right],
\end{gathered}
$$


where $(x, z)$ are the horizontal/vertical position of a particle that is originally at a horizontal position $\alpha$ along the material curve given by $\delta=$ constant. On the water free surface, the stress components vanish (assuming that the free surface is clean and air is inviscid):

$$
T_{w}=0, \quad N_{w}=0 \quad \text { on } \delta=h_{w} .
$$

Along the upper/lower fluid interface, there is continuity of fluid particle displacements

$$
x_{w}=x_{m}, \quad z_{w}=z_{m} \quad \text { on } \delta=0,
$$

and continuity of stress components

$$
T_{w}=T_{m}, \quad N_{w}=N_{m} \quad \text { on } \delta=0 .
$$

Suppose the bed is rigid and impermeable, and hence there will not be any fluid motion

$$
x_{m}=z_{m}=0 \quad \text { on } \delta=-h_{m} .
$$

On the basis of small-amplitude displacements, the variables may be expanded as follows (Pierson 1962):

$$
\begin{gathered}
\left(x_{f}, z_{f}\right)=(\alpha, \delta)+\epsilon\left(x_{f}^{(1)}, z_{f}^{(1)}\right)+\epsilon^{2}\left(x_{f}^{(2)}, z_{f}^{(2)}\right)+\cdots, \\
p_{f}=\rho_{w} g h_{w}-\rho_{f} g \delta+\epsilon p_{f}^{(1)}+\epsilon^{2} p_{f}^{(2)}+\cdots .
\end{gathered}
$$

Perturbation equations are obtainable for the $O(\epsilon)$ and $O\left(\epsilon^{2}\right)$ variables on substituting (2.16) and (2.17) into the Lagrangian equations above and collecting terms of equal powers of $\epsilon$.

\section{First-order problem}

At $O(\epsilon)$, the equations of motion (2.3), (2.5) and (2.6) yield

$$
\begin{gathered}
\frac{\partial x_{f}^{(1)}}{\partial \alpha}+\frac{\partial z_{f}^{(1)}}{\partial \delta}=0, \\
\ddot{x}_{f}^{(1)}=-\frac{1}{\rho_{f}} \frac{\partial p_{f}^{(1)}}{\partial \alpha}-g \frac{\partial z_{f}^{(1)}}{\partial \alpha}+v_{f} \frac{\partial^{2} \dot{x}_{f}^{(1)}}{\partial \delta^{2}}, \\
0=-\frac{1}{\rho_{f}} \frac{\partial p_{f}^{(1)}}{\partial \delta}-g \frac{\partial z_{f}^{(1)}}{\partial \delta}
\end{gathered}
$$

where $v_{f}=\mu_{f} / \rho_{f}$ is the kinematic viscosity of the fluid. On the free surface, the kinematic boundary condition requires $z_{w}^{(1)}\left(\alpha, \delta=h_{w}, t\right)$ to be equal to $\eta_{w}(\alpha, t)$ given by (2.1). Also, the dynamic free-surface boundary conditions (2.12) give

$$
\frac{\partial \dot{x}_{w}^{(1)}}{\partial \delta}=0, \quad p_{w}^{(1)}=0 \quad \text { on } \delta=h_{w} .
$$

Along the upper/lower fluid interface, the matching conditions (2.13) and (2.14) give

$$
\begin{gathered}
x_{w}^{(1)}=x_{m}^{(1)}, \quad z_{w}^{(1)}=z_{m}^{(1)} \quad \text { on } \delta=0, \\
\mu_{w} \frac{\partial \dot{x}_{w}^{(1)}}{\partial \delta}=\mu_{m} \frac{\partial \dot{x}_{m}^{(1)}}{\partial \delta}, \quad p_{w}^{(1)}=p_{m}^{(1)} \quad \text { on } \delta=0 .
\end{gathered}
$$

At the bottom the boundary conditions are simply $\dot{x}_{m}^{(1)}=\dot{z}_{m}^{(1)}=0$ on $\delta=-h_{m}$. 
Solutions to this first-order problem can be found by first separating the variables into components due to the forward and backward propagating waves:

$$
\left(x_{f}^{(1)}, z_{f}^{(1)}, p_{f}^{(1)}\right)=\operatorname{Re}\left[\left(\tilde{x}_{f}, \tilde{z}_{f}, \tilde{p}_{f}\right) \mathrm{e}^{\mathrm{i}(k \alpha-\sigma t)}+R\left(-\tilde{x}_{f}^{*}, \tilde{z}_{f}^{*}, \tilde{p}_{f}^{*}\right) \mathrm{e}^{\mathrm{i}\left(k^{*} \alpha+\sigma t\right)}\right],
$$

where $\tilde{x}, \tilde{z}$ and $\tilde{p}$ are complex functions of $\delta$. Now, following the steps of Dalrymple \& Liu (1978), who have solved a similar two-layer problem, we may express the solutions as follows. For $h_{w}>\delta>0$ (i.e. in the water layer),

$$
\begin{gathered}
\tilde{z}_{w}(\delta)=A \delta+B+C \exp \left[\beta_{w}\left(\delta-h_{w}\right)\right]+D \exp \left(-\beta_{w} \delta\right), \\
\tilde{x}_{w}(\delta)=\mathrm{i} k^{-1}\left\{A+\beta_{w} C \exp \left[\beta_{w}\left(\delta-h_{w}\right)\right]-\beta_{w} D \exp \left(-\beta_{w} \delta\right)\right\} .
\end{gathered}
$$

For $0>\delta>-h_{m}$ (i.e. in the lower viscous layer),

$$
\begin{gathered}
\tilde{z}_{m}(\delta)=G\left[\sinh \beta_{m}\left(\delta+h_{m}\right)-\beta_{m}\left(\delta+h_{m}\right)\right]+H\left[\cosh \beta_{m}\left(\delta+h_{m}\right)-1\right], \\
\tilde{x}_{m}(\delta)=\mathrm{i} k^{-1} \beta_{m}\left\{G\left[\cosh \beta_{m}\left(\delta+h_{m}\right)-1\right]+H \sinh \beta_{m}\left(\delta+h_{m}\right)\right\} .
\end{gathered}
$$

In these expressions,

$$
\beta_{f}=(1-\mathrm{i}) / s_{f}
$$

where $s_{f}=\left(2 v_{f} / \sigma\right)^{1 / 2}$ is the Stokes boundary layer thickness in the respective fluid. Also, on substituting into the free-surface and interface boundary conditions, the complex constants $A-D, G$ and $H$ are found to be

$$
\begin{gathered}
A=g k^{2} a / \sigma^{2}, \\
B=\left(1-g k^{2} h_{w} / \sigma^{2}\right) a, \\
C=0, \\
D=I^{-1}\left\{\left(\beta_{m} h_{m} \cosh \beta_{m} h_{m}-\sinh \beta_{m} h_{m}\right) A+\beta_{m}\left(1-\cosh \beta_{m} h_{m}\right) B\right\} \\
G=I^{-1}\left\{\left(-\cosh \beta_{m} h_{m}+\gamma \cosh \beta_{m} h_{m}-\gamma\right) A-\left(\gamma \beta_{m} \sinh \beta_{m} h_{m}+\beta_{w} \cosh \beta_{m} h_{m}\right) B\right\}, \\
\qquad \begin{array}{c}
-1\left\{\left(\sinh \beta_{m} h_{m}-\gamma \sinh \beta_{m} h_{m}+\gamma \beta_{m} h_{m}\right) A\right. \\
\left.+\left[\beta_{w} \sinh \beta_{m} h_{m}+\gamma \beta_{m}\left(-1+\cosh \beta_{m} h_{m}\right)\right] B\right\}
\end{array} \\
\text { where } \gamma=\rho_{w} / \rho_{m} \text { is the ratio of the fluid densities, and } \\
I=\beta_{m}(2 \gamma-1)\left(1-\cosh \beta_{m} h_{m}\right)+\gamma \beta_{m}^{2} h_{m} \sinh \beta_{m} h_{m} \\
\quad+\beta_{w}\left(\beta_{m} h_{m} \cosh \beta_{m} h_{m}-\sinh \beta_{m} h_{m}\right)
\end{gathered}
$$

In addition, the wavenumber $k$ is found to satisfy the following dispersion relation:

$$
\begin{aligned}
& \left(\frac{\sigma^{2}}{g k^{2}}\right)^{2}\left\{\gamma \beta_{m}^{2} \sinh \beta_{m} h_{m}+\beta_{w} \beta_{m} \cosh \beta_{m} h_{m}\right\} \\
& \quad+\left(\frac{\sigma^{2}}{g k^{2}}\right)\left\{\beta_{m} \cosh \beta_{m} h_{m}+\beta_{w} \sinh \beta_{m} h_{m}-\beta_{w} \beta_{m} h \cosh \beta_{m} h_{m}-\gamma \beta_{m}^{2} h \sinh \beta_{m} h_{m}\right\} \\
& \quad+(1-\gamma)\left\{\left(\sinh \beta_{m} h_{m}-\beta_{m} h_{m} \cosh \beta_{m} h_{m}\right)\left(1-\beta_{w} h_{w}\right)\right. \\
& \left.\quad+\gamma \beta_{m}^{2} h_{m} h_{w} \sinh \beta_{m} h_{m}+2 \gamma \beta_{m} h_{w}\left(1-\cosh \beta_{m} h_{m}\right)\right\}=0
\end{aligned}
$$

where $h=h_{w}+h_{m}$ is the total fluid depth. 
The solutions presented above deserve some remarks. First, it has been assumed that the ratio of the Stokes boundary layer thickness to the respective fluid depth $s_{f} / h_{f}$ is a finite fraction of unity for the lower viscous layer, but is much smaller than unity for the water layer. In other words, the viscosity is important, to the first order, throughout the entire lower viscous layer, but only near the free surface and the interface in the water layer. Therefore, hyperbolic functions are used to express the viscous parts of the solutions for the lower viscous layer, but for the water layer only the exponential terms that decay far from the boundaries are considered. The exponential terms in (3.8) and (3.9) that are multiplied by $C$ and $D$ are respectively significant only within the free surface and the interface boundary layers. It however turns out that, under the long-wave limit, the constant $C$ is zero, implying that a boundary layer does not need to exist in order to satisfy the vanishing of shear stress at the free surface. Second, on invoking the assumption that $s_{w} \ll s_{m}$ or $\left|\beta_{w}\right| \gg\left|\beta_{m}\right|$, the dispersion relation (3.20) can be simplified to give the following solution for the wavenumber:

$$
\begin{aligned}
\frac{\sigma^{2}}{g h k^{2}}= & \frac{1}{2}\left\{1-\frac{\tanh \beta_{m} h_{m}}{\beta_{m} h}\right. \\
& \left.-\left[\left(\frac{\tanh \beta_{m} h_{m}}{\beta_{m} h}-1\right)^{2}+4(1-\gamma)\left(\frac{\tanh \beta_{m} h_{m}}{\beta_{m} h}-\frac{h_{m}}{h}\right) \frac{h_{w}}{h}\right]^{1 / 2}\right\},
\end{aligned}
$$

where the principal value of the square root is to be used. To estimate the order of magnitude of the real and imaginary parts of the wavenumber $k=k_{r}+\mathrm{i} k_{i}$, we may further suppose that the lower-layer viscous fluid is not much denser than water $(\gamma \approx 1)$ and the Stokes boundary layer thickness in the viscous fluid is comparable to the fluid depth $\left(s_{m} \approx h_{m}\right)$, by which the solution above can be simplified to

$$
k(g h)^{1 / 2} / \sigma \sim 1+(0.34+0.20 \mathrm{i}) s_{m} / h .
$$

It is then clear that the imaginary part of $k\left(k_{i}(g h)^{1 / 2} / \sigma \sim 10^{-2}\right)$ can be much smaller than the real part of $k\left(k_{r}(g h)^{1 / 2} / \sigma \sim 1\right)$ as long as $s_{m}$ remains a small fraction of $h$ (say, $<0.2$ ). These results are confirmed by an example shown in figure 2 , in which values of $k_{r}(g h)^{1 / 2} / \sigma$ and $k_{i}(g h)^{1 / 2} / \sigma$ have been calculated from the dispersion relation (3.20) for some typical values of the fluid properties $\left(\gamma=0.8, s_{w} / h=0.001, s_{m} / h=0.2,0.1\right.$, 0.01 , and $\left.0<h_{m} / h<0.5\right)$. In this example case, it is found that $1<k_{r}(g h)^{1 / 2} / \sigma<1.08$ and $0<k_{i}(g h)^{1 / 2} / \sigma<0.06$. Therefore, while the wave celerity is approximately given by the classical long-wave value (i.e. $\left.c \equiv \sigma / k_{r}=(g h)^{1 / 2}\right)$, the wave decay will not be significant until a distance much longer than a wavelength is considered. Since in the present work our attention is limited to a horizontal length comparable to a wavelength, we may ignore the wave attenuation, or drop $k_{i}$ from $k$. This is justifiable as long as $k_{i} / k_{r} \leqslant O(\epsilon)$ so that the omission of $k_{i}$ from $k$ will only lead to error terms of higher orders. From here on, we shall not distinguish $k_{r}$ from $k$, which is simply taken as a real quantity.

\section{Second-order problem}

The $O\left(\epsilon^{2}\right)$ governing equations are as follows:

$$
\frac{\partial x_{f}^{(2)}}{\partial \alpha}+\frac{\partial z_{f}^{(2)}}{\partial \delta}=-\frac{\partial x_{f}^{(1)}}{\partial \alpha} \frac{\partial z_{f}^{(1)}}{\partial \delta}+\frac{\partial x_{f}^{(1)}}{\partial \delta} \frac{\partial z_{f}^{(1)}}{\partial \alpha},
$$



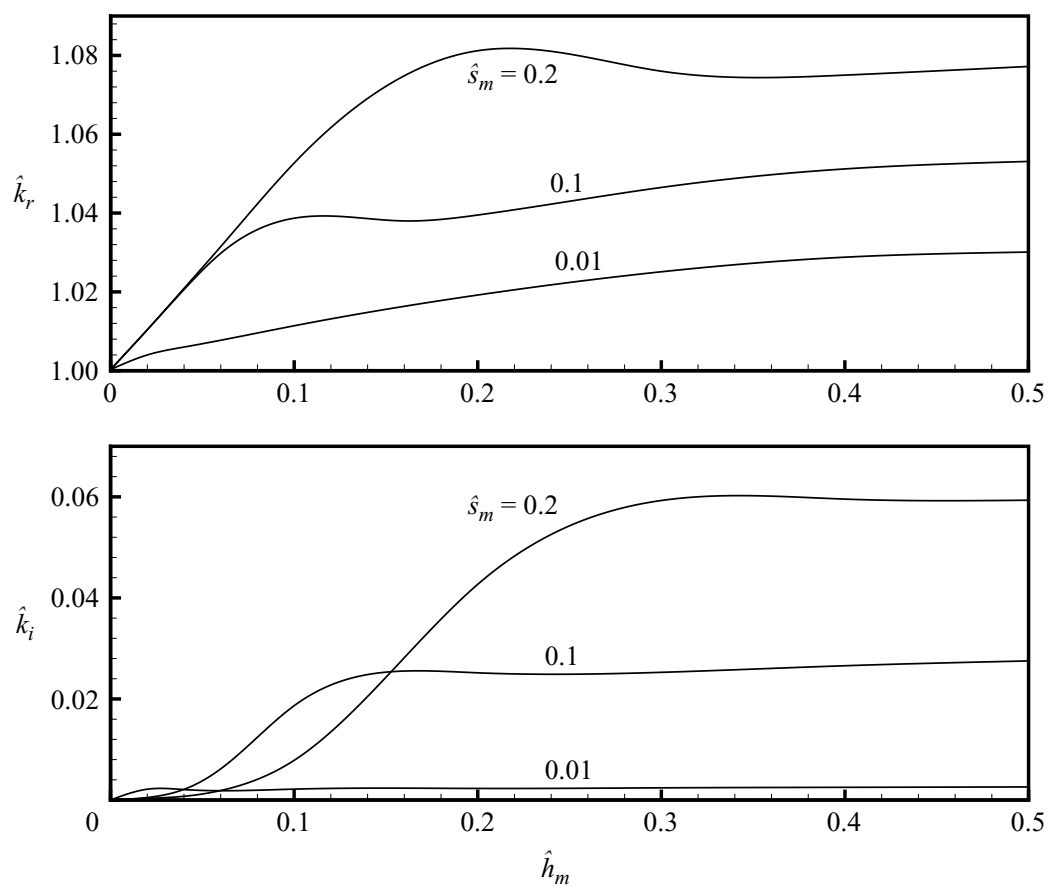

FIGURE 2. The real and imaginary parts of the wavenumber, $\hat{k}_{r}=k_{r}(g h)^{1 / 2} / \sigma$ and $\hat{k}_{i}=$ $k_{i}(g h)^{1 / 2} / \sigma$, as functions of the lower-layer fluid depth $\hat{h}_{m}=h_{m} / h$ and the Stokes boundary layer thickness $\hat{s}_{m}=s_{m} / h$.

$$
\begin{gathered}
\ddot{x}_{f}^{(2)}+\frac{1}{\rho_{f}} \frac{\partial p_{f}^{(2)}}{\partial \alpha}+g \frac{\partial z_{f}^{(2)}}{\partial \alpha}-v_{f} \frac{\partial^{2} \dot{x}_{f}^{(2)}}{\partial \delta^{2}}=X_{f}^{(2)}, \\
\frac{1}{\rho_{f}} \frac{\partial p_{f}^{(2)}}{\partial \delta}+g \frac{\partial z_{f}^{(2)}}{\partial \delta}=Z_{f}^{(2)},
\end{gathered}
$$

where the forcing terms

$$
\begin{gathered}
X_{f}^{(2)}=-\ddot{x}_{f}^{(1)} \frac{\partial x_{f}^{(1)}}{\partial \alpha}+v_{f}\left[3 \frac{\partial^{2} \dot{x}_{f}^{(1)}}{\partial \delta^{2}} \frac{\partial x_{f}^{(1)}}{\partial \alpha}-2 \frac{\partial^{2} \dot{x}_{f}^{(1)}}{\partial \alpha \partial \delta} \frac{\partial x_{f}^{(1)}}{\partial \delta}-\frac{\partial \dot{x}_{f}^{(1)}}{\partial \alpha} \frac{\partial^{2} x_{f}^{(1)}}{\partial \delta^{2}}+\frac{\partial \dot{x}_{f}^{(1)}}{\partial \delta} \frac{\partial^{2} x_{f}^{(1)}}{\partial \alpha \partial \delta}\right], \\
Z_{f}^{(2)}=-\ddot{x}_{f}^{(1)} \frac{\partial x_{f}^{(1)}}{\partial \delta}+v_{f} \frac{\partial^{2} \dot{x}_{f}^{(1)}}{\partial \delta^{2}} \frac{\partial x_{f}^{(1)}}{\partial \delta}
\end{gathered}
$$

consist of products of the first-order variables. On the free surface of the water layer, the dynamic boundary conditions give

$$
\begin{gathered}
\frac{\partial \dot{x}_{w}^{(2)}}{\partial \delta}=-3 \frac{\partial \dot{x}_{w}^{(1)}}{\partial \delta} \frac{\partial x_{w}^{(1)}}{\partial \alpha}+\frac{\partial \dot{x}_{w}^{(1)}}{\partial \alpha} \frac{\partial x_{w}^{(1)}}{\partial \delta} \quad \text { on } \delta=h_{w}, \\
p_{w}^{(2)}=-2 p_{w}^{(1)} \frac{\partial x_{w}^{(1)}}{\partial \alpha} \quad \text { on } \delta=h_{w} .
\end{gathered}
$$

Along the upper/lower fluid interface, the matching conditions are

$$
x_{w}^{(2)}=x_{m}^{(2)}, \quad z_{w}^{(2)}=z_{m}^{(2)} \quad \text { on } \delta=0,
$$




$$
\begin{gathered}
\mu_{w}\left(\frac{\partial \dot{x}_{w}^{(2)}}{\partial \delta}+3 \frac{\partial \dot{x}_{w}^{(1)}}{\partial \delta} \frac{\partial x_{w}^{(1)}}{\partial \alpha}-\frac{\partial \dot{x}_{w}^{(1)}}{\partial \alpha} \frac{\partial x_{w}^{(1)}}{\partial \delta}\right) \\
=\mu_{m}\left(\frac{\partial \dot{x}_{m}^{(2)}}{\partial \delta}+3 \frac{\partial \dot{x}_{m}^{(1)}}{\partial \delta} \frac{\partial x_{m}^{(1)}}{\partial \alpha}-\frac{\partial \dot{x}_{m}^{(1)}}{\partial \alpha} \frac{\partial x_{m}^{(1)}}{\partial \delta}\right) \quad \text { on } \delta=0 \\
p_{w}^{(2)}+2 p_{w}^{(1)} \frac{\partial x_{w}^{(1)}}{\partial \alpha}=p_{m}^{(2)}+2 p_{m}^{(1)} \frac{\partial x_{m}^{(1)}}{\partial \alpha} \quad \text { on } \delta=0 .
\end{gathered}
$$

On substituting the first-order solutions, (4.6) and (4.7) are simplified to

$$
\frac{\partial \dot{x}_{w}^{(2)}}{\partial \delta}=0, \quad p_{w}^{(2)}=0 \quad \text { on } \delta=h_{w},
$$

while (4.9) and (4.10) also reduce to

$$
\mu_{w} \frac{\partial \dot{x}_{w}^{(2)}}{\partial \delta}=\mu_{m} \frac{\partial \dot{x}_{m}^{(2)}}{\partial \delta}, \quad p_{w}^{(2)}=p_{m}^{(2)} \quad \text { on } \delta=0 .
$$

At the bottom the boundary conditions are $\dot{x}_{m}^{(2)}=\dot{z}_{m}^{(2)}=0$ on $\delta=-h_{m}$.

The mass transport velocity is defined to be the steady component of the $O\left(\epsilon^{2}\right)$ Lagrangian drift:

$$
\left(u_{L f}, w_{L f}\right) \equiv\left(\overline{\dot{x}}_{f}^{(2)}, \overline{\dot{z}}_{f}^{(2)}\right),
$$

where the overbar denotes time average over a period. As noted by Piedra-Cueva (1995), the continuity equation for the mass transport velocity is obtainable as follows by first differentiating (4.1) with respect to time followed by time-averaging over a period:

$$
\frac{\partial u_{L f}}{\partial \alpha}+\frac{\partial w_{L f}}{\partial \delta}=0 .
$$

By integrating this equation with respect to $\delta$ across the individual fluid layers, we may further obtain

$$
\begin{gathered}
\frac{\partial Q_{w}}{\partial \alpha}+\frac{\partial \bar{\eta}_{w}^{(2)}}{\partial t}-\frac{\partial \bar{\eta}_{m}^{(2)}}{\partial t}=0, \\
\frac{\partial Q_{m}}{\partial \alpha}+\frac{\partial \bar{\eta}_{m}^{(2)}}{\partial t}=0
\end{gathered}
$$

where

$$
Q_{w}=\int_{0}^{h_{w}} u_{L w} \mathrm{~d} \delta, \quad Q_{m}=\int_{-h_{m}}^{0} u_{L m} \mathrm{~d} \delta
$$

are the discharges due to the mass transport velocity in the water and viscous fluid layers, and

$$
\bar{\eta}_{w}^{(2)}=\left.\bar{z}_{w}^{(2)}\right|_{\delta=h_{w}}, \quad \bar{\eta}_{m}^{(2)}=\left.\bar{z}_{w}^{(2)}\right|_{\delta=0}=\left.\bar{z}_{m}^{(2)}\right|_{\delta=0}
$$

are the Lagrangian set-ups (i.e. the steady mean displacement) of the water free surface and the upper/lower fluid interface. Equations (4.15) and (4.16) govern the slow time evolution of the set-ups as a result of any non-uniform spatial distribution of the discharges. $\mathrm{Ng} \& \mathrm{Fu}$ (2002) have shown that it takes a time scale that is one order of magnitude longer than the wave period for a set-up to evolve into the equilibrium profile. Now, let us consider that long enough time has passed so that a steady second-order field of Lagrangian mean motion is established and the set-ups 
are fully developed. On reaching this stage, (4.15) and (4.16) become

$$
\frac{\partial Q_{w}}{\partial \alpha}=0, \quad \frac{\partial Q_{m}}{\partial \alpha}=0,
$$

or the discharges must not depend on $\alpha$ any longer. These conditions will be utilized later to find the free surface and the interfacial set-ups.

We next take the time average of (4.3), which is then integrated with respect to $\delta$. For the water and viscous fluid layers, the equation yields respectively

$$
\frac{1}{\rho_{w}} \bar{p}_{w}^{(2)}+g \bar{z}_{w}^{(2)}=g \bar{\eta}_{w}^{(2)}+\int_{h_{w}}^{\delta} \bar{Z}_{w}^{(2)} \mathrm{d} \delta \quad \text { for } h_{w}>\delta>0,
$$

and

$$
\begin{aligned}
\frac{1}{\rho_{m}} \bar{p}_{m}^{(2)}+g \bar{z}_{m}^{(2)}=\gamma g \bar{\eta}_{w}^{(2)} & +(1-\gamma) g \bar{\eta}_{m}^{(2)} \\
+\gamma \int_{h_{w}}^{0} \bar{Z}_{w}^{(2)} \mathrm{d} \delta+\int_{0}^{\delta} \bar{Z}_{m}^{(2)} \mathrm{d} \delta \quad \text { for } 0>\delta>-h_{m}, &
\end{aligned}
$$

where the free-surface and interface boundary conditions for $\bar{p}^{(2)}$ have been used.

\subsection{Mass transport in the water layer}

\subsubsection{Free-surface set-up}

Substituting (4.20) and the first-order solutions into the time-averaged (4.2), we get the governing equation for the mass transport velocity in the water layer:

$$
\begin{aligned}
v_{w} \frac{\partial^{2} u_{L w}}{\partial \delta^{2}}= & g \frac{\partial \bar{\eta}_{w}^{(2)}}{\partial \alpha}+\left(1-R^{2}\right) \sigma^{2} k^{-1}\left\{-2 A \operatorname{Im}\left[\beta_{w} D \exp \left(-\beta_{w} \delta\right)\right]+\frac{3}{2}\left|\beta_{w} D\right|^{2} \exp \left(-2 \beta_{w r} \delta\right)\right\} \\
& +R \sigma^{2} k^{-1} \sin 2 k \alpha\left\{-A^{2}-4 A \operatorname{Re}\left[\beta_{w} D \exp \left(-\beta_{w} \delta\right)\right]\right. \\
& \left.+3\left|\beta_{w} D\right|^{2} \exp \left(-2 \beta_{w r} \delta\right)\right\} \quad \text { for } h_{w}>\delta>0
\end{aligned}
$$

where $\beta_{w r}$ is the real part of $\beta_{w}$. In the interior region well above the interface boundary layer, the equation reduces to

$$
v_{w} \frac{\partial^{2} u_{L w}}{\partial \delta^{2}}=g \frac{\partial \bar{\eta}_{w}^{(2)}}{\partial \alpha}-R \sigma^{2} k^{-1} A^{2} \sin 2 k \alpha .
$$

One can readily check that the viscous diffusion term on the left-hand side is of $O\left(s_{w} / h\right)^{2}$, much smaller than the non-viscous term on the right-hand side of the equation. Therefore, to balance these two terms of vastly different orders of magnitude, we need to accordingly separate the free surface set-up into the non-viscous and viscous parts

$$
\bar{\eta}_{w}^{(2)}=\bar{\eta}_{w N}^{(2)}+\bar{\eta}_{w V}^{(2)}
$$

where $\bar{\eta}_{w V}^{(2)} / \bar{\eta}_{w N}^{(2)}=O\left(s_{w} / h\right)^{2}$, such that

$$
g \frac{\partial \bar{\eta}_{w N}^{(2)}}{\partial \alpha}=R \sigma^{2} k^{-1} A^{2} \sin 2 k \alpha
$$

and

$$
v_{w} \frac{\partial^{2} u_{L w}}{\partial \delta^{2}}=g \frac{\partial \bar{\eta}_{w V}^{(2)}}{\partial \alpha}
$$


Integrating (4.25) gives us an expression for the free-surface set-up due to the inviscid part of the wave motion:

$$
\bar{\eta}_{w N}^{(2)}=-\frac{R g k^{2} a^{2}}{2 \sigma^{2}} \cos 2 k \alpha+\text { constant }
$$

where (3.13) has been substituted for $A$. In the particular case when the wave is purely standing $(R=1.0)$ and the system is a single layer of inviscid fluid such that $\sigma^{2}=g k^{2} h$, the Lagrangian free-surface set-up given above becomes

$$
\bar{\eta}_{w N}^{(2)}=-\frac{a^{2}}{2 h} \cos 2 k \alpha+\text { constant },
$$

which apparently is different by a sign from the classical Eulerian expression derived by Longuet-Higgins \& Stewart (1964):

$$
\bar{\zeta}=\frac{a^{2}}{2 h} \cos 2 k \alpha
$$

This apparent difference can be reconciled by noting that, in general, the Lagrangian description $\eta$ of the displacement of a material surface $\delta=\delta_{I}$ is not exactly the same as its Eulerian description $\zeta$. A relation has been deduced by $\mathrm{Ng}(2004 a)$ between these two quantities when the displacement of the material surface is small in amplitude, as in the present case. In terms of the time-averaged second-order displacements, the relation is

$$
\bar{\eta}^{(2)}=\bar{\zeta}^{(2)}+\overline{\left(x^{(1)} \frac{\partial z^{(1)}}{\partial \alpha}\right)_{\delta_{I}}},
$$

where we have denoted $z\left(\delta=\delta_{I}\right)$ by $\eta(\alpha, t)$.

For the present case, one can evaluate the second term on the right-hand side of (4.30) to be, for $\delta=h_{w}$ and $R=1.0$,

$$
\overline{\left(x_{w}^{(1)} \frac{\partial z_{w}^{(1)}}{\partial \alpha}\right)_{\delta=h_{w}}}=\frac{a^{2}}{h}(1-\cos 2 k \alpha) .
$$

Adding this to (4.29) gives

$$
\bar{\zeta}+\overline{\left(x_{w}^{(1)} \frac{\partial z_{w}^{(1)}}{\partial \alpha}\right)_{\delta=h_{w}}}=-\frac{a^{2}}{2 h} \cos 2 k \alpha+\frac{a^{2}}{h},
$$

which matches $\bar{\eta}_{w N}^{(2)}$ given in (4.28) provided that the constant is equal to $a^{2} / h$. Therefore, by this exercise the integration constant is also fixed, and (4.27) may now be written as

$$
\bar{\eta}_{w N}^{(2)}=\frac{R g k^{2} a^{2}}{\sigma^{2}}\left[-\frac{1}{2} \cos 2 k \alpha+1\right] .
$$

It is interesting to further note that even though the Eulerian set-up $\bar{\zeta}$ has a zero spatial mean, the corresponding Lagrangian set-up $\bar{\eta}_{w N}^{(2)}$ does not satisfy this condition. This kind of difference, namely the Lagrangian-mean elevation of a wave being higher than a line cutting off equal areas, has been noted and explained before by McIntyre (1988). It is essentially due to the fact that the Lagrangian average of a surface elevation is evenly weighted over the initial positions of the surface particles rather than their displaced positions. See McIntyre (1988, especially figure 1) and the references therein for further details. 
We may now subtract the non-viscous terms from (4.22), which is then solved in turn for the mass transport velocity in the interface boundary layer and in the core region.

\subsubsection{Interface boundary layer}

In the water boundary layer near the interface, the free-surface set-up term $g \partial \bar{\eta}_{w V}^{(2)} /$ $\partial \alpha$ is subdominant and can be ignored. Integrating (4.22) twice with respect to $\delta$ gives

$$
\begin{aligned}
u_{L w}(\alpha, \delta)= & u_{L I}+\left(1-R^{2}\right) \sigma k^{-1}\left\{-2 A \operatorname{Re}\left[\beta_{w} D\left(\exp \left(-\beta_{w} \delta\right)-1\right)\right]\right. \\
& \left.+\frac{3}{4}\left|\beta_{w} D\right|^{2}\left(\exp \left(-2 \beta_{w r} \delta\right)-1\right)\right\}+R \sigma k^{-1} \sin 2 k \alpha\left\{4 A \operatorname{Im}\left[\beta_{w} D\left(\exp \left(-\beta_{w} \delta\right)-1\right)\right]\right. \\
& \left.+\frac{3}{2}\left|\beta_{w} D\right|^{2}\left(\exp \left(-2 \beta_{w r} \delta\right)-1\right)\right\} \quad \text { for } \delta=O\left(s_{w}\right)>0,
\end{aligned}
$$

where $u_{L I}=\left.u_{L w}\right|_{\delta=0}$ is the interfacial value of $u_{L w}$, which will be matched with the interfacial value of $u_{L m}$ to be given in (4.50). At a distance far above the interface, the above solution tends to the following limit:

$$
\begin{aligned}
u_{L w}\left(\alpha, h_{w} \gg \delta \gg s_{w}\right)=u_{L I} & +\left(1-R^{2}\right) \sigma k^{-1}\left\{2 A \operatorname{Re}\left(\beta_{w} D\right)-\frac{3}{4}\left|\beta_{w} D\right|^{2}\right\} \\
& -R \sigma k^{-1} \sin 2 k \alpha\left\{4 A \operatorname{Im}\left(\beta_{w} D\right)+\frac{3}{2}\left|\beta_{w} D\right|^{2}\right\},
\end{aligned}
$$

which provides a bottom boundary condition for the solution in the core region. Unlike the one-layer case, this mass transport velocity at the outer edge of the boundary layer depends on the viscosity of the fluid.

\subsubsection{Core region}

Equation (4.26) governs the mass transport in the water region that is well above the interface boundary layer, subject to the condition $\partial u_{L w} / \partial \delta=0$ at the top and (4.35) at the bottom. The solution can readily be found to be

$$
\begin{aligned}
u_{L w}(\alpha, \delta)= & \delta\left(\frac{\delta}{2}-h_{w}\right) \frac{g}{v_{w}} \frac{\partial \bar{\eta}_{w V}^{(2)}}{\partial \alpha}+\left(1-R^{2}\right)\left\{\sigma k^{-1}\left[2 A \operatorname{Re}\left(\beta_{w} D\right)-\frac{3}{4}\left|\beta_{w} D\right|^{2}\right]+u_{L I D}\right\} \\
& -R \sin 2 k \alpha\left\{\sigma k^{-1}\left[4 A \operatorname{Im}\left(\beta_{w} D\right)+\frac{3}{2}\left|\beta_{w} D\right|^{2}\right]-u_{L I A}\right\} \quad \text { for } h_{w}>\delta>O\left(s_{w}\right),
\end{aligned}
$$

where $u_{L I}$ has been separated into the unidirectional and alternating parts

$$
u_{L I}=\left(1-R^{2}\right) u_{L I D}+(R \sin 2 k \alpha) u_{L I A},
$$

where, according to (4.50) below,

$$
\begin{gathered}
u_{L I D}=\frac{\sigma^{2}}{v_{m} k}\left[\left.U_{m D}\right|_{\delta=0}-Y \frac{3}{2 h_{m}} Q_{m D}\right], \\
u_{L I A}=\frac{\sigma^{2}}{v_{m} k}\left[\left.U_{m A}\right|_{\delta=0}-\frac{3}{2 h_{m}} Q_{m A}\right],
\end{gathered}
$$

in which $Y$ is an integer parameter to be defined in (4.42), and expressions for $U_{m D}$, $U_{m A}, Q_{m D}$ and $Q_{m A}$, which are to be introduced in (4.46) and (4.47), are given in the Appendix. By (4.17), the discharge in the water layer is given by

$$
\begin{array}{r}
Q_{w}=-\frac{g h_{w}^{3}}{3 v_{w}} \frac{\partial \bar{\eta}_{w V}^{(2)}}{\partial \alpha}+\left(1-R^{2}\right)\left\{\sigma k^{-1}\left[2 A \operatorname{Re}\left(\beta_{w} D\right)-\frac{3}{4}\left|\beta_{w} D\right|^{2}\right]+u_{L I D}\right\} h_{w} \\
-R \sin 2 k \alpha\left\{\sigma k^{-1}\left[4 A \operatorname{Im}\left(\beta_{w} D\right)+\frac{3}{2}\left|\beta_{w} D\right|^{2}\right]-u_{L I A}\right\} h_{w} .
\end{array}
$$


If the domain is bounded at its far end, the net discharge must vanish or $Q_{w}=0$. If not, the discharge $Q_{w}$ is still a constant according to (4.19). At this point, we need to distinguish between the cases of an open and a closed system. By a reflected wave in an open system, we shall mean that the point of interest is in the middle of a large but finite domain, and the reflected wave has reached the point but the return current is not felt yet. This is possible because the propagation speed of the wave is two orders of magnitude faster than the return current. The vertical profile of second-order mean motions can also be established well before the arrival of the return current (Ünlüata \& Mei 1970). Now, taking either far-end condition of the system into account, the viscous part of the free-surface set-up may be set to satisfy

$$
\begin{aligned}
\frac{g h_{w}^{2}}{3 v_{w}} \frac{\partial \bar{\eta}_{w V}^{(2)}}{\partial \alpha}=Y(1- & \left.R^{2}\right)\left\{\sigma k^{-1}\left[2 A \operatorname{Re}\left(\beta_{w} D\right)-\frac{3}{4}\left|\beta_{w} D\right|^{2}\right]+u_{L I D}\right\} \\
& -R \sin 2 k \alpha\left\{\sigma k^{-1}\left[4 A \operatorname{Im}\left(\beta_{w} D\right)+\frac{3}{2}\left|\beta_{w} D\right|^{2}\right]-u_{L I A}\right\},
\end{aligned}
$$

where

$$
Y= \begin{cases}0 & \text { if the domain is open, } \\ 1 & \text { if the domain is closed. }\end{cases}
$$

We remark that for a closed domain a return current, which induces the first part of the set-up in (4.41), must be generated in order to balance the unidirectional part of the mass transport so that the total discharge vanishes. For an open domain, the return current does not exist and the discharge is due only to the second term in the first line of (4.40). The second part of the set-up in (4.41), which is induced by the alternating part of the mass transport, does not depend on whether the domain is bounded or not; mass transport in a pure standing wave will form recirculating cells and therefore the discharge is always zero when the set-up is fully developed. We also remark that, on comparing (4.25) and (4.41), $\bar{\eta}_{w V}^{(2)}$ is indeed smaller than $\bar{\eta}_{w N}^{(2)}$ by of order $\left(s_{w} / h\right)^{2}$. Despite its smallness in magnitude, this viscous part of the free-surface set-up must be taken into account in order to satisfy the conservation of mass.

Putting (4.41) back into (4.36), we finally arrive at an expression for the horizontal mass transport velocity in the water core region:

$$
\begin{aligned}
& u_{L w}(\alpha, \delta)= {\left[1+Y \frac{3 \delta}{h_{w}^{2}}\left(\frac{\delta}{2}-h_{w}\right)\right]\left(1-R^{2}\right)\left\{\sigma k^{-1}\left[2 A \operatorname{Re}\left(\beta_{w} D\right)-\frac{3}{4}\left|\beta_{w} D\right|^{2}\right]+u_{L I D}\right\} } \\
&-\left[1+\frac{3 \delta}{h_{w}^{2}}\left(\frac{\delta}{2}-h_{w}\right)\right] R \sin 2 k \alpha\left\{\sigma k^{-1}\left[4 A \operatorname{Im}\left(\beta_{w} D\right)+\frac{3}{2}\left|\beta_{w} D\right|^{2}\right]-u_{L I A}\right\}, \\
& \text { for } h_{w}>\delta>O\left(s_{w}\right) .
\end{aligned}
$$

The vertical mass transport velocity in the water core region is then found by integrating the continuity equation (4.14):

$$
w_{L w}=w_{L I}-\int_{0}^{\delta} \frac{\partial u_{L w}}{\partial \alpha} \mathrm{d} \delta,
$$

where $w_{L I}=w_{L w}(\delta=0)=w_{L m}(\delta=0)$ can be determined from (4.51) below.

\subsection{Mass transport in the lower-layer viscous fluid}

The substitution of (4.21) into the time-averaged (4.2), where $\bar{\eta}_{w}^{(2)}$ is replaced by $\bar{\eta}_{w N}^{(2)}$, yields the following equation after some algebra:

$$
v_{m} \frac{\partial^{2} u_{L m}}{\partial \delta^{2}}=(1-\gamma) g \frac{\partial \bar{\eta}_{m}^{(2)}}{\partial \alpha}+\left(1-R^{2}\right) \sigma^{2} k^{-1} f_{D}+R \sigma^{2} k^{-1}(\sin 2 k \alpha) f_{A},
$$


(III)

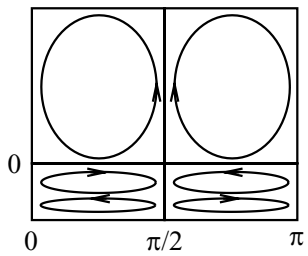

(II)

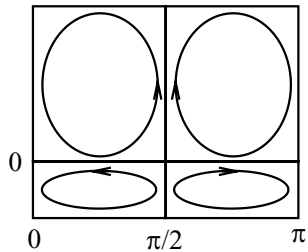

(I)

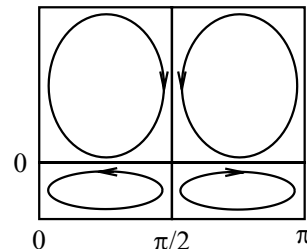

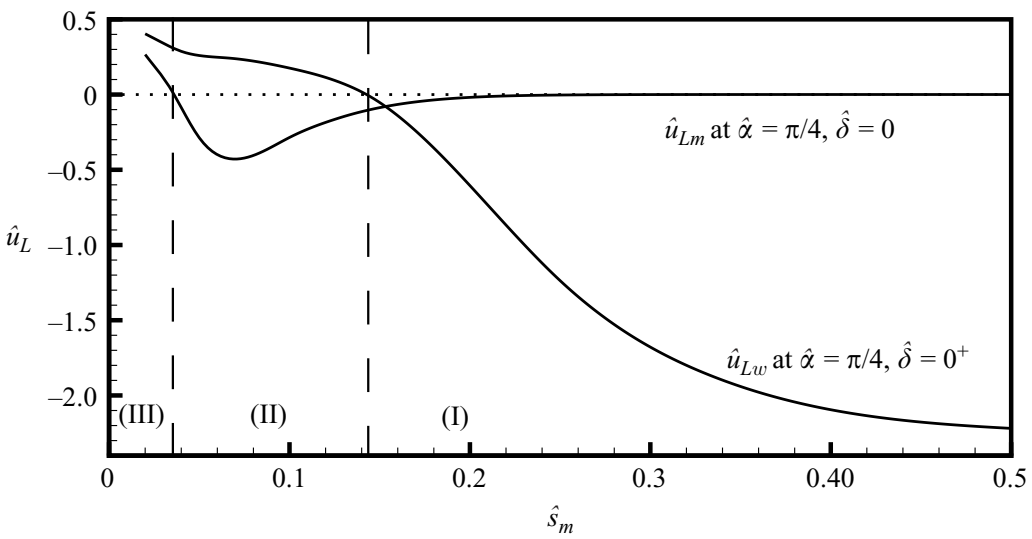

FIGURE 3. The maximum mass transport velocity in the upper-layer water core region $\hat{u}_{L w}$ and that in the lower-layer viscous fluid $\hat{u}_{L m}$, which occur at $\hat{\alpha}=\pi / 4$ immediately above and below the interface $\hat{\delta}=0$, as functions of the Stokes boundary layer thickness $\hat{s}_{m}$ when under a pure standing wave $(R=1.0)$; three patterns of recirculation cells I, II, and III are shown schematically according to the sign of these mass transport velocities.

where $f_{D}$ and $f_{A}$ are real functions of $\delta$, which are detailed in the Appendix. Integrating this equation twice with respect to $\delta$, using the boundary conditions that $v_{m} \partial u_{L m} /$ $\partial \delta=\gamma v_{w} \partial u_{L w} / \partial \delta$ at $\delta=0$ and $u_{L m}=0$ at $\delta=-h_{m}$, we obtain

$$
u_{L m}(\alpha, \delta)=\left(\delta^{2}-h_{m}^{2}\right)(1-\gamma) \frac{g}{2 v_{m}} \frac{\partial \bar{\eta}_{m}^{(2)}}{\partial \alpha}+\left(1-R^{2}\right) \frac{\sigma^{2}}{v_{m} k} U_{m D}+R \frac{\sigma^{2}}{v_{m} k}(\sin 2 k \alpha) U_{m A},
$$

where $U_{m D}(\delta)$ and $U_{m A}(\delta)$ are also given in the Appendix. Putting this into (4.17) gives an expression for the discharge in the lower viscous layer:

$$
Q_{m}=-(1-\gamma) \frac{g h_{m}^{3}}{3 v_{m}} \frac{\partial \bar{\eta}_{m}^{(2)}}{\partial \alpha}+\left(1-R^{2}\right) \frac{\sigma^{2}}{v_{m} k} Q_{m D}+R \frac{\sigma^{2}}{v_{m} k}(\sin 2 k \alpha) Q_{m A},
$$

where $Q_{m D}$ and $Q_{m A}$ are two constants, whose expressions are also provided in the Appendix. Again, by the conservation-of-mass condition (4.19), the interfacial set-up must satisfy

$$
(1-\gamma) \frac{g h_{m}^{3}}{3 v_{m}} \frac{\partial \bar{\eta}_{m}^{(2)}}{\partial \alpha}=Y\left(1-R^{2}\right) \frac{\sigma^{2}}{v_{m} k} Q_{m D}+R \frac{\sigma^{2}}{v_{m} k}(\sin 2 k \alpha) Q_{m A},
$$

where $Y$, which is an integer switching parameter as defined in (4.42), is inserted to encompass two possible far-end conditions of the domain. From (4.48), it can be checked that the interfacial set-up is of order $\left(s_{m} / h\right)^{2}$ compared with that of the nonviscous free-surface set-up. Therefore, since it has been supposed that $s_{m}$ is a finite fraction of the lower fluid depth, the three set-ups are related to one another as 
(III)

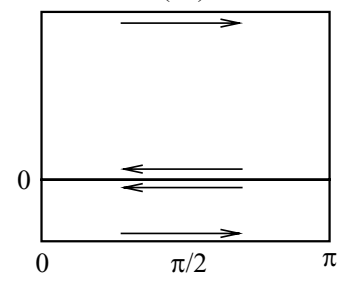

(II)

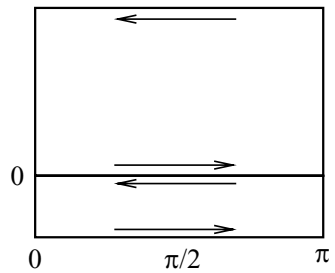

(I)

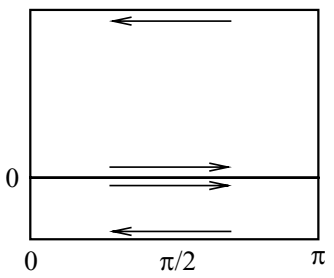

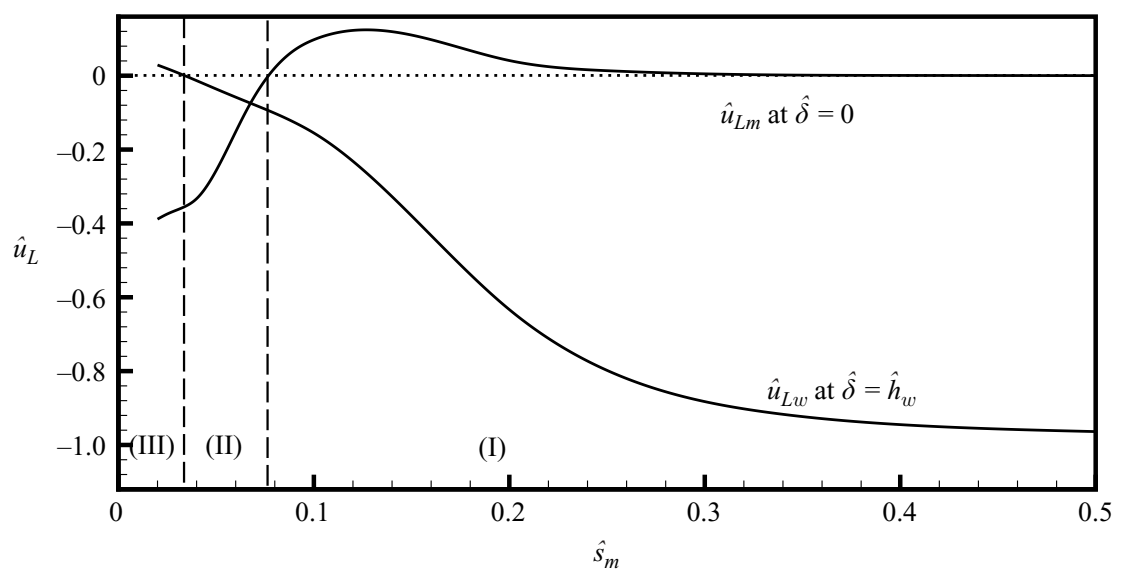

FIGURE 4. The mass transport velocity of the upper-layer water at the free surface $\hat{u}_{L w}\left(\hat{\delta}=\hat{h}_{w}\right)$, and that of the lower-layer viscous fluid at the interface $\hat{u}_{L m}(\hat{\delta}=0)$, as functions of the Stokes boundary layer thickness $\hat{s}_{m}$ when under a pure progressive wave in a closed system $(R=0$, $Y=1$ ); three patterns of drift profiles I, II, and III are shown schematically according to the sign of these mass transport velocities.

follows:

$$
\bar{\eta}_{w N}^{(2)}>\bar{\eta}_{m}^{(2)} \gg \bar{\eta}_{w V}^{(2)}
$$

Putting (4.48) back into (4.46), we finally obtain an expression for the horizontal mass transport velocity in the lower viscous layer:

$$
\begin{aligned}
u_{L m}(\alpha, \delta)=\left(1-R^{2}\right) \frac{\sigma^{2}}{v_{m} k}[ & \left.U_{m D}+Y \frac{3}{2 h_{m}^{3}} Q_{m D}\left(\delta^{2}-h_{m}^{2}\right)\right] \\
& +R \frac{\sigma^{2}}{v_{m} k} \sin 2 k \alpha\left[U_{m A}+\frac{3}{2 h_{m}^{3}} Q_{m A}\left(\delta^{2}-h_{m}^{2}\right)\right] .
\end{aligned}
$$

The vertical mass transport velocity in the lower viscous layer is then found from

$$
w_{L m}=-\int_{-h_{m}}^{\delta} \frac{\partial u_{L m}}{\partial \alpha} \mathrm{d} \delta .
$$

\section{Results and discussion}

Numerical calculations have been performed in terms of the following normalized variables, which are distinguished by a caret:

$$
\left.\begin{array}{rr}
\alpha=k^{-1} \hat{\alpha}, & \left(\delta, h_{w}, h_{m}, s_{w}, s_{m}\right)=h\left(\hat{\delta}, \hat{h}_{w}, \hat{h}_{m}, \hat{s}_{w}, \hat{s}_{m}\right), \\
t=\sigma^{-1} \hat{t} \quad\left(u_{L f}, w_{L f}, Q_{f}\right) & =(a / h)^{2}(\sigma / k)\left(\hat{u}_{L f}, k h \hat{w}_{L f}, h \hat{Q}_{f}\right) .
\end{array}\right\}
$$



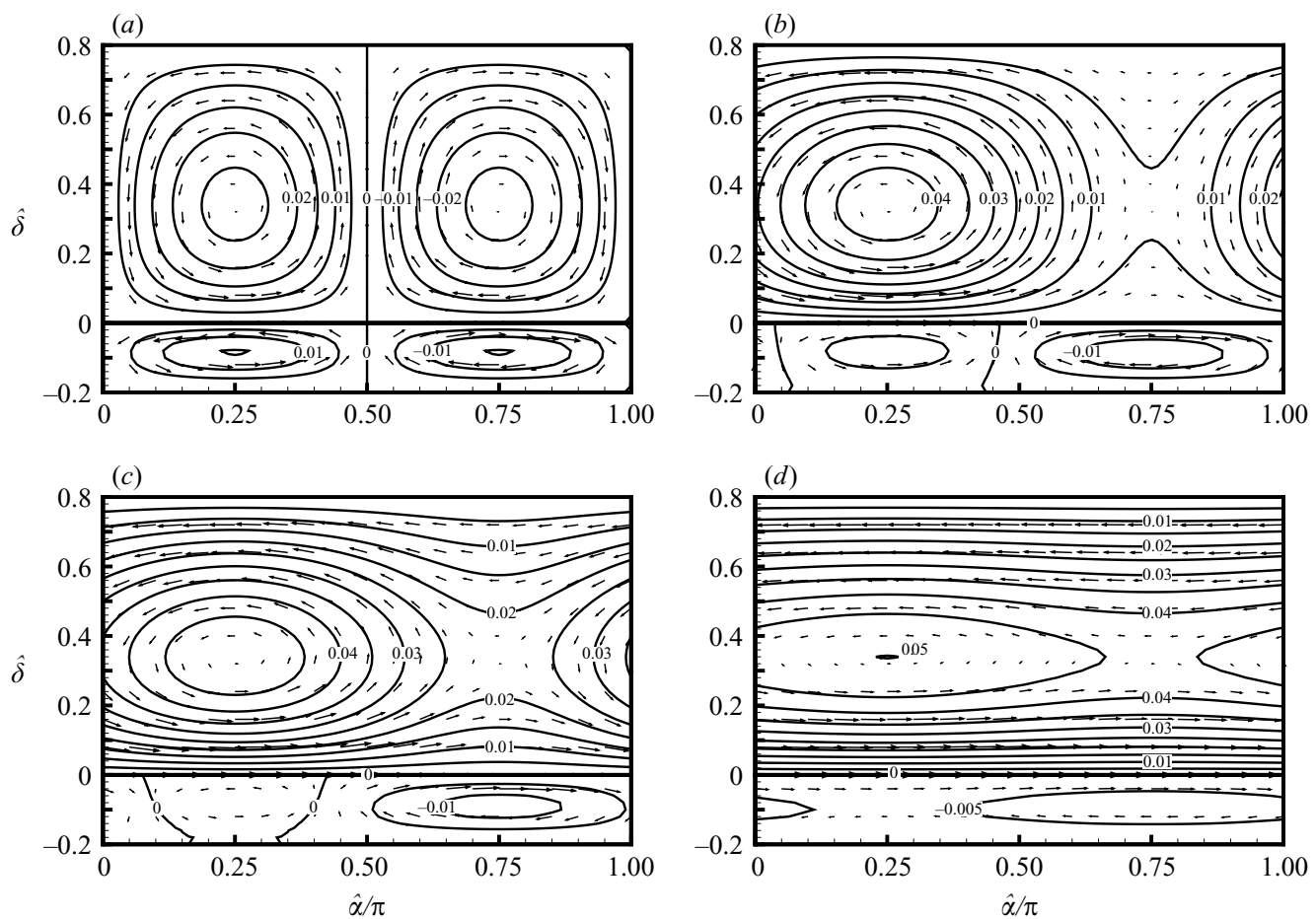

Figure 5. Mass transport streamlines and velocity vectors in partially standing waves in a closed system $(Y=1)$ for $\hat{s}_{m}=0.1$, and $(a) R=1.0,(b) R=0.7,(c) R=0.5,(d) R=0.1$.

The mass transport velocity components and other quantities can be evaluated upon specifying the following input parameters: density ratio $(\gamma)$, depth of the lower layer $\left(\hat{h}_{m}\right)$, Stokes boundary layer thicknesses in water $\left(\hat{s}_{w}\right)$ and in the lower-layer viscous fluid $\left(\hat{s}_{m}\right)$, reflection coefficient $(R)$, and switching parameter for the far-end condition of the domain $(Y)$. We are here primarily interested in examining the effects of $\hat{s}_{m}$, $R$ and $Y$ on the mass transport flow field, and have fixed the values of other inputs in the calculations as follows: $\gamma=0.8, \hat{s}_{w}=10^{-3}$ and $\hat{h}_{m}=0.2$. Also, comparisons are made between two particular values of the viscous fluid viscosity: $\hat{s}_{m}=0.1$ and 0.02 . The higher value of $\hat{s}_{m}$ corresponds to a highly viscous fluid, which is so viscous that the Stokes boundary layer thickness is large and equal to half the fluid-layer depth. The lower value of $\hat{s}_{m}$ corresponds to a less viscous fluid, and the Stokes boundary layer thickness is only one tenth of the fluid-layer depth. With the chosen fixed $\hat{s}_{w}=10^{-3}$, the two values of $\hat{s}_{m}=0.1$ and 0.02 also correspond to $\left(v_{m} / v_{w}\right)^{1 / 2}=100$ and 20 respectively. Muds of viscosity varying over such a range of values have been used in the experiments by Sakakiyama \& Bijker (1989), and considered in the computations by Piedra-Cueva (1995).

Let us first examine the mass transport in the limiting case of a pure standing wave $(R=1.0)$, for which whether the system is closed or not is immaterial. The mass transport is known to exhibit a periodic cellular structure with a circulation cell formed over a quarter of the wavelength between a node and an antinode of the free surface. In the present problem, the antinodes are at $\hat{\alpha}=n \pi$, and the nodes are at $\hat{\alpha}=(2 n+1) \pi / 2$, where $n=0, \pm 1, \ldots$ Figure 3 shows how the rotation of the cells formed between $\hat{\alpha}=0$ and $\pi$ in the two layers will vary with the viscosity of the lower-layer fluid. Plotted in this graph are the maximum mass transport velocities 

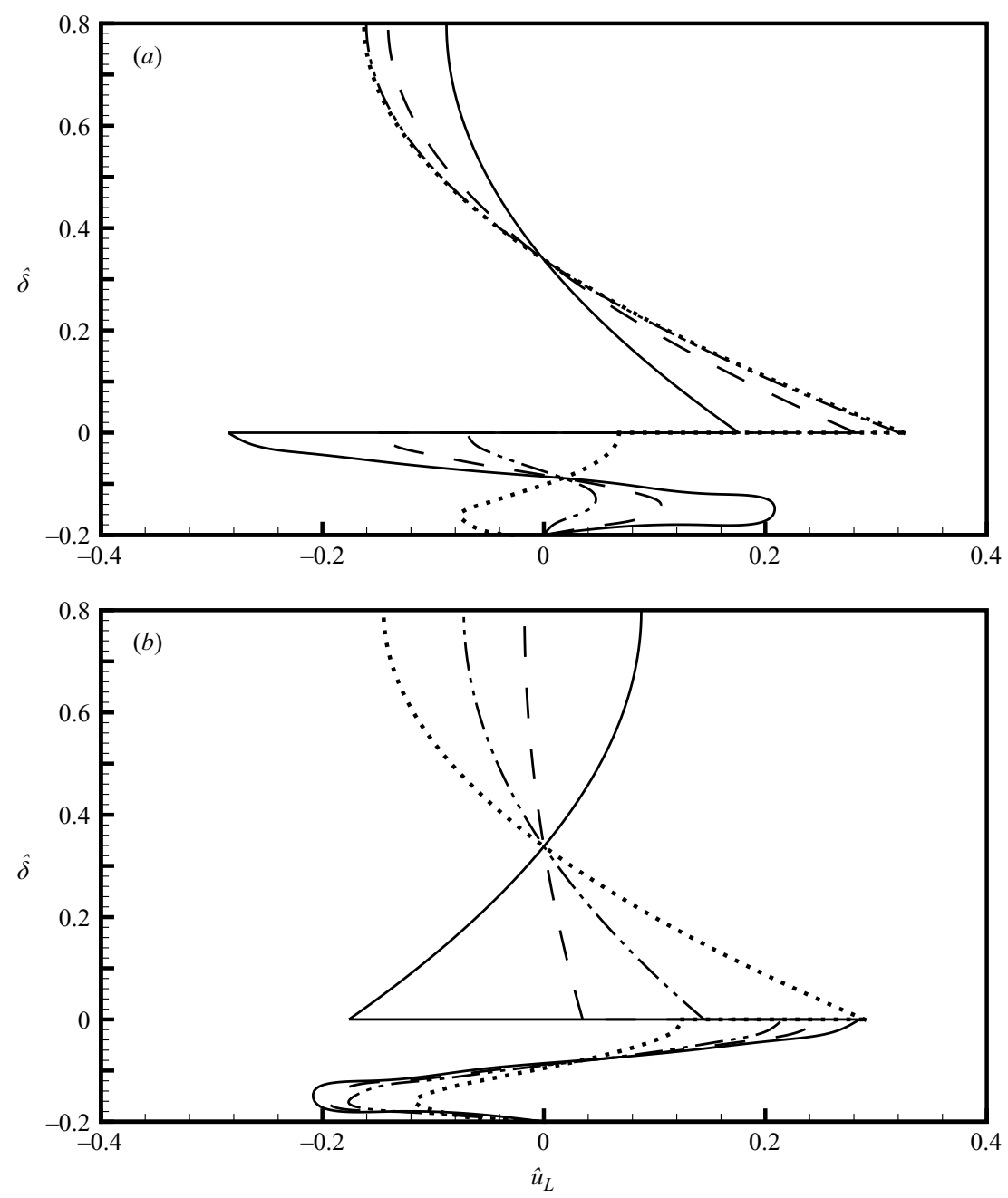

FIGURE 6 . Mass transport velocity profiles at $(a) \hat{\alpha}=\pi / 4,(b) \hat{\alpha}=3 \pi / 4$ under reflected waves in a closed system $(Y=1)$ for $\hat{s}_{m}=0.1(-, R=1.0 ;-\longrightarrow, R=0.7 ;-\cdots-, R=5 ; \cdots, R=0.1)$.

in the left-hand cells, which occur immediately above and below the interface in the water core region and in the lower layer at $\hat{\alpha}=\pi / 4$. These mass transport velocities give not only the strength of the circulation, but also the direction of rotation of the cells in the two layers. The flow patterns can be broadly divided into three cases. Case I is when the lower fluid is so highly viscous that it hardly flows under the wave excitation. The lower layer becomes virtually a rigid bed when $\hat{s}_{m}>0.5$, and the classical case of a single layer (Longuet-Higgins 1953) is then recovered. In this limiting case of a single fluid, the left-hand cell rotates clockwise, and a thin jet shoots upwards under an antinode. The flow in the lower layer is not appreciable until $\hat{s}_{m}<0.2$. On reaching this stage, an anticlockwise cell forms on the left-hand side (i.e. $0<\hat{\alpha}<\pi / 2)$ in the lower layer, and a thin jet shoots downwards under an antinode in the lower fluid. It is remarkable that decreasing the viscosity of the lower fluid not only diminishes the mass transport velocity of the upper fluid, but also eventually reverses the rotation of the cells in the upper layer. This happens in this particular 

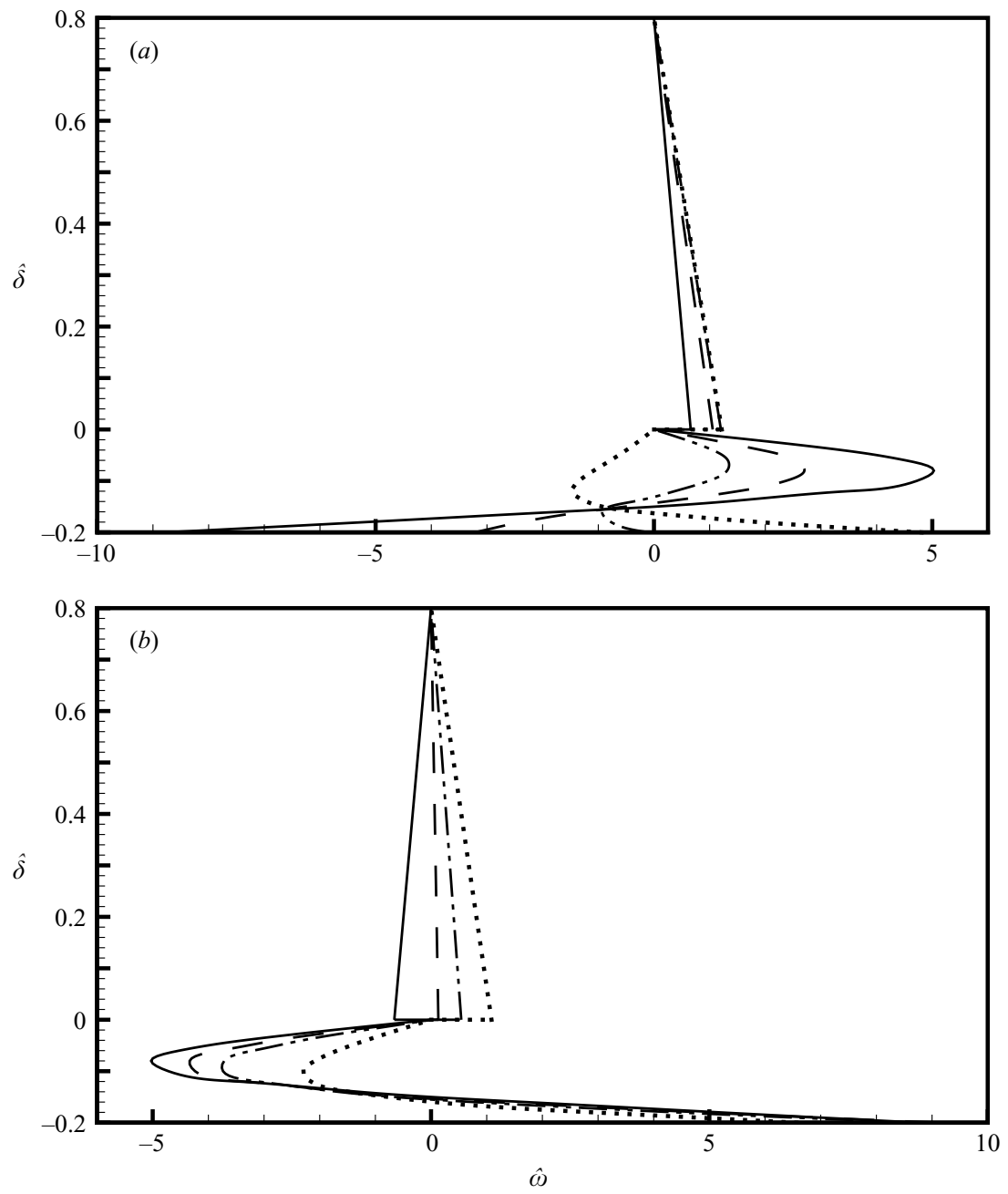

FiguRE 7. Vorticity profiles at $(a) \hat{\alpha}=\pi / 4,(b) \hat{\alpha}=3 \pi / 4$ under reflected waves in a closed system $(Y=1)$ for $\hat{s}_{m}=0.1(-, R=1.0 ;-\longrightarrow, R=0.7 ;-\cdots-, R=5 ; \cdots, R=0.1)$.

example when $\hat{s}_{m}=0.142$. It is interesting to note that at this threshold value of $\hat{s}_{m}$ the mass transport field vanishes everywhere in the core region of the upper layer under a pure standing wave. In case II, when $\hat{s}_{m}$ is smaller than the threshold value, the left-hand cell in each layer is anticlockwise, resulting in thin vertical jets shooting upwards, now under a node in both fluids. The cell patterns for $\hat{s}_{m}=0.1$ are shown in figure $5(a)$. On further decreasing the lower-fluid viscosity so that $s_{m}$ then becomes only a small fraction of the fluid depth, a boundary layer structure also emerges near the bottom of the lower layer. In case III, when $\hat{s}_{m}<0.035$, two distinct cells, one on top of the other, now appear between a node and an antinode in the lower layer. Figure $8(a)$ shows such a recirculation structure at $\hat{s}_{m}=0.02$. In this case, the lower cell is already much weakened and confined near the bottom. This occurrence of vertical jet-like motions directed upwards/downwards in the upper/lower fluid in the neighbourhood of a nodal position is consistent with the analytical findings of Dore (1973). 

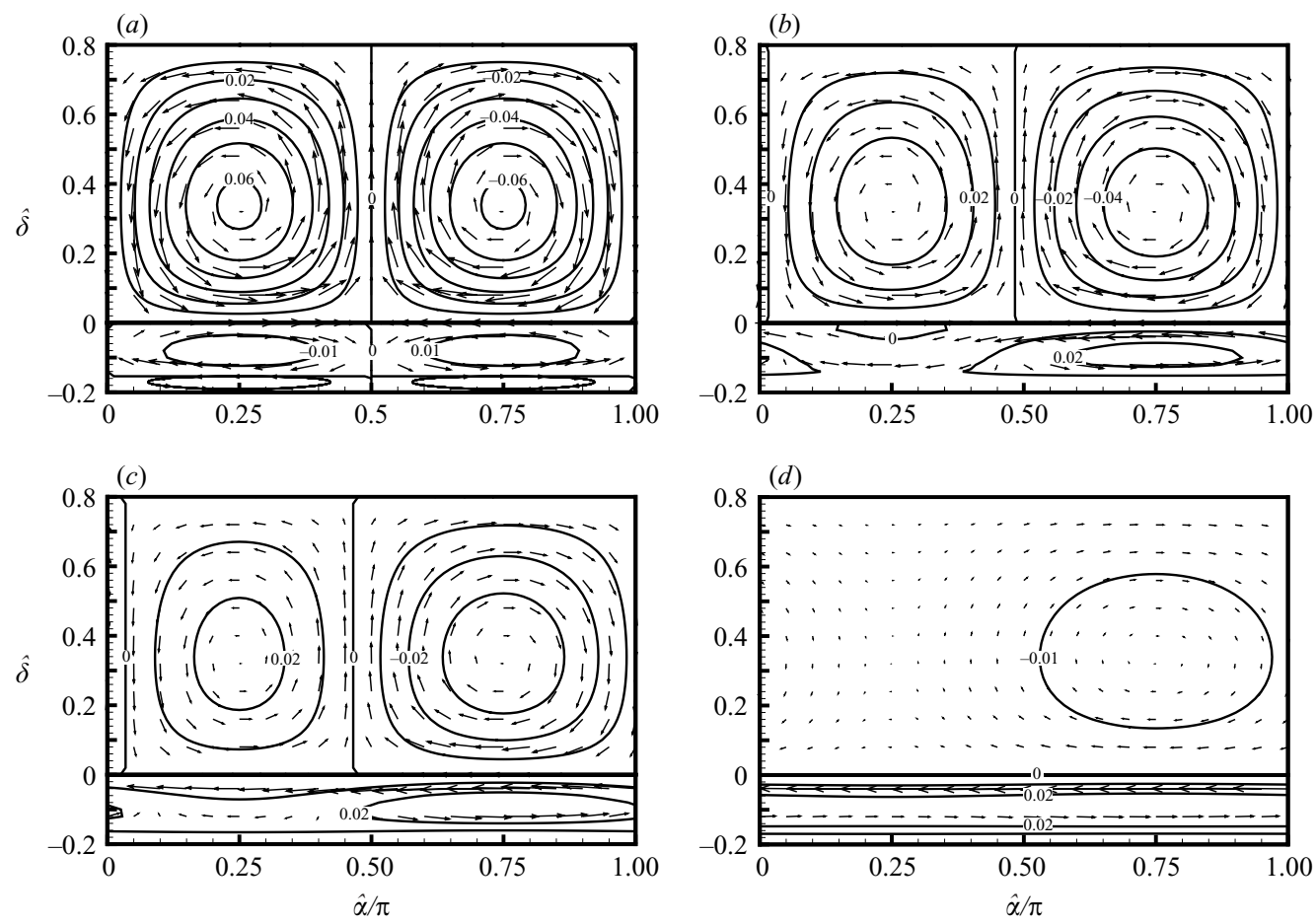

FIGURE 8. Mass transport streamlines and velocity vectors in partially standing waves in a closed system $(Y=1)$ for $\hat{s}_{m}=0.02$, and (a) $R=1.0,(b) R=0.7,(c) R=0.5,(d) R=0.1$.

We next examine the other limiting case, namely a pure progressive wave $(R=0)$, when the system is closed $(Y=1)$. The mass transport is strictly horizontal in a progressive wave, but because of the return current the velocity will turn from positive to negative across part of a section in order to maintain a zero total flow in each layer. Based on the specific experimental conditions of Sakakiyama \& Bijker (1989), Piedra-Cueva (1995) has performed calculations in terms of physical quantities and found that for long waves the mass transport velocities at the free surface and the interface may be positive or negative depending on the viscosity of the lower-layer fluid. We here look into the relationships in a more general manner. Figure 4 shows the dependence on $\hat{s}_{m}$ of the interfacial mass transport velocity $\left.\hat{u}_{L m}\right|_{\hat{\delta}=0}$ and the mass transport velocity on the water free surface $\hat{u}_{L w} \mid \hat{\delta}_{\hat{\delta}} \hat{h}_{w}$. We may infer from the sign of these velocities the change in direction of the drift profiles across the two layers. As shown in the figure, three possible cases corresponding to different ranges of $\hat{s}_{m}$ may again be classified. Case I again covers the case when the lower layer is virtually rigid, for which the classical behaviour for a long wave (Longuet-Higgins 1953), namely a strong forward velocity near the bottom and a milder backward velocity near the free surface, is exhibited in the upper layer. More precisely, at such a limit the backward velocity at the free surface is half the magnitude of the forward velocity at the bottom of the layer. Decreasing the lower-layer viscosity will tend to decrease/increase the velocity of flow in the upper/lower layer. In the lower layer, the drifts near the interface and near the bottom will reverse their directions as $\hat{s}_{m}$ crosses the value of 0.075. It is remarkable that in case II the drift is backward immediately below the interface in the lower layer, but is forward near the interface in the upper layer. This 

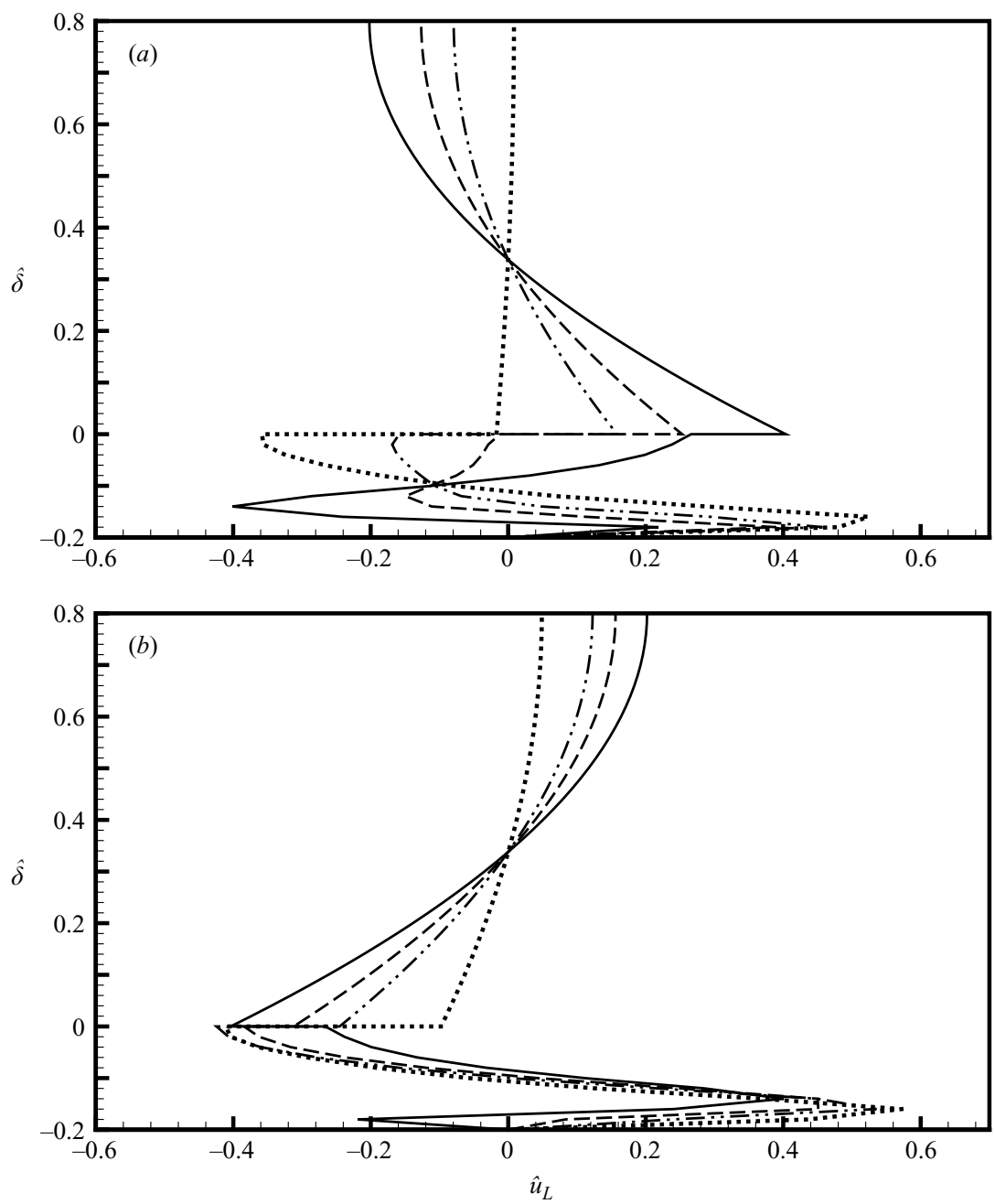

FiguRE 9. Mass transport velocity profiles at $(a) \hat{\alpha}=\pi / 4$, (b) $\hat{\alpha}=3 \pi / 4$ under reflected waves in a closed system $(Y=1)$ for $\hat{s}_{m}=0.02(-, R=1.0 ;-\square, R=0.7 ;-\cdots-, R=5 ; \cdots$, $R=0.1$ ).

implies a strong velocity gradient in the boundary layer above the interface. The flow in the upper layer is further weakened until it becomes completely quiescent when $\hat{s}_{m}$ decreases to the value of 0.035 . This upper-bound value for the present case III happens to be exactly the same as the one for the previous case III discussed in the preceding paragraph (see figure 3 ). On crossing over to case III, while the flow remains weak, the drift in the upper layer changes to become forward near the free surface and backward near the interface.

From here on, let us examine in greater detail the various flow patterns associated with the two particular values of $\hat{s}_{m}$ mentioned above. We show in figure 5 the Lagrangian streamlines and velocity vectors for a range of the reflection coefficient when $\hat{s}_{m}=0.1$ and the domain is closed. This value of $\hat{s}_{m}$ leads to a case II configuration defined in figure 3 when under a standing wave, and a case I configuration defined in figure 4 when under a progressive wave. Also note that the water boundary 

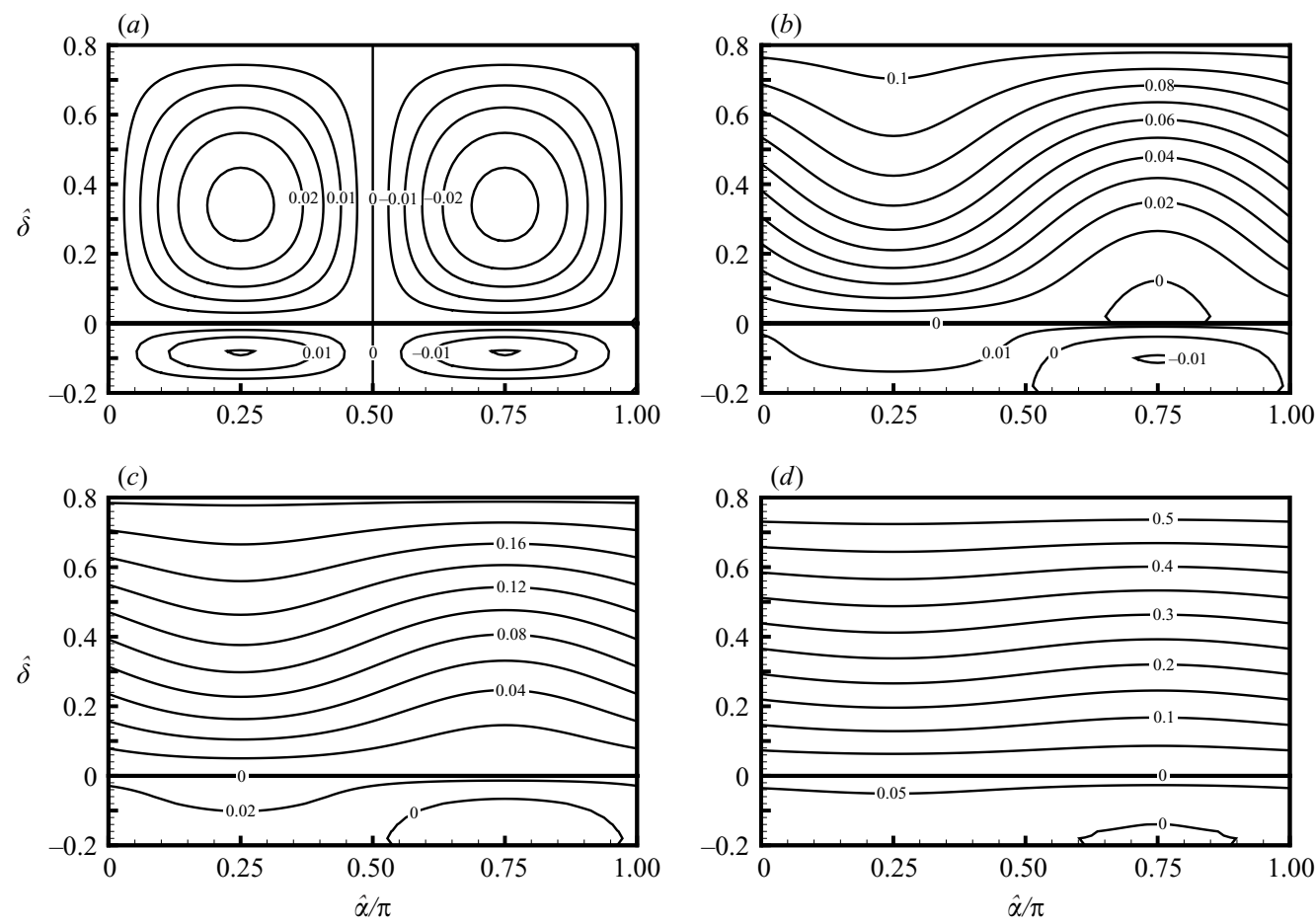

FIGURE 10. Mass transport streamlines in partially standing waves in an open system $(Y=0)$ for $\hat{s}_{m}=0.1$, and (a) $R=1.0,(b) R=0.95,(c) R=0.9,(d) R=0.7$.

layer near the interface, which is very small in thickness, has been omitted in this and the other figures. This explains why it appears that the velocity is not continuous across the interface. For this highly viscous fluid, an obvious effect of decreasing $R$ (i.e. a smaller amplitude of the reflected wave) is to cause the right-hand cell in the water layer and the left-hand cell in the lower layer to disappear or to lose form first. The unidirectional (forward and reverse) currents tend to enhance the left-hand cell, but to counteract the right-hand cell in the water layer. As a result, the recirculation in the left-hand cell will increase in strength and size with a decreasing $R$ until a significant forward/backward drift has developed below/above the recirculation. The recirculation in each layer continues to exist however, even when $R$ is as low as 0.1. The corresponding vertical profiles of the mass transport velocity and the vorticity at $\hat{\alpha}=\pi / 4$ and $3 \pi / 4$ are shown respectively in figures 6 and 7 . The latter figure illustrates that the vorticity in the lower viscous layer is in general much higher than that in the water core region. The vorticity is typically maximum at the bottom of the lower layer.

Figure 8 shows the streamlines and velocity vectors for the case of $\hat{s}_{m}=0.02$ when the domain is also closed. For this less viscous fluid, decreasing the value of $R$ will weaken both cells (the left-hand one being more affected) in the water layer, but will strengthen the right-hand cell in the lower layer. The cells in the water layer can retain their identity however, even when $R$ drops below 0.5 . According to figure 4 , this value of $\hat{s}_{m}$ leads to a case III configuration when the progressive wave limit is approached. Indeed it is noticeable that for a low reflection coefficient $(R \leqslant 0.1)$, the flow field in the water layer is so weak that it is almost quiescent. 

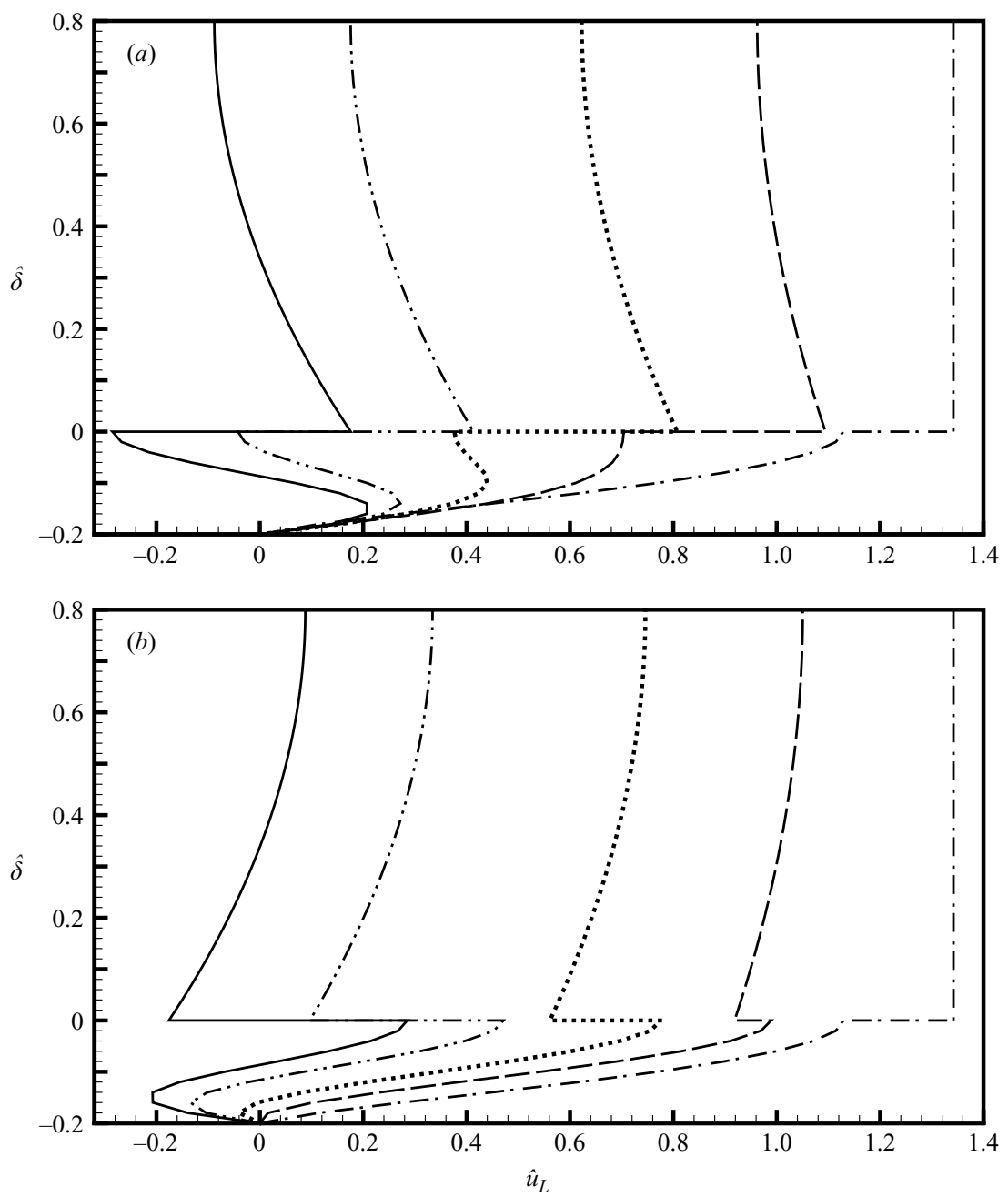

FiguRE 11. Mass transport velocity profiles at $(a) \hat{\alpha}=\pi / 4,(b) \hat{\alpha}=3 \pi / 4$ under reflected waves in an open system $(Y=0)$ for $\hat{s}_{m}=0.1(\longleftarrow, R=1.0 ;-\cdots-R=0.9 ; \cdots, R=0.7 ;-\square$, $R=0.5 ;-\cdot-, R=0.0)$.

This lack of mass transport in the water layer happens despite the occurrence of a rather strong drift near the interface and a sharp shear flow in the lower layer (figure 9).

The flow fields for $\hat{s}_{m}=0.1$ and 0.02 when the domain is open-ended are shown respectively in figures 10 and 12 , while the corresponding velocity profiles are respectively given in figures 11 and 13. In the absence of return currents, the circulation cells are readily eliminated by a slight deviation of $R$ from unity. In the case of the higher fluid viscosity (figure 10), the flow in the water layer will become mostly unidirectional when $R$ drops only by $10 \%$. In the case of the lower-fluid viscosity (figure 12), the merging of the two cells as $R$ is slightly reduced to 0.95 results in an interesting flow pattern in the water layer: a strong and thick jet passes under the left-hand cell and shoots upwards to pass over the right-hand cell and then shoots 

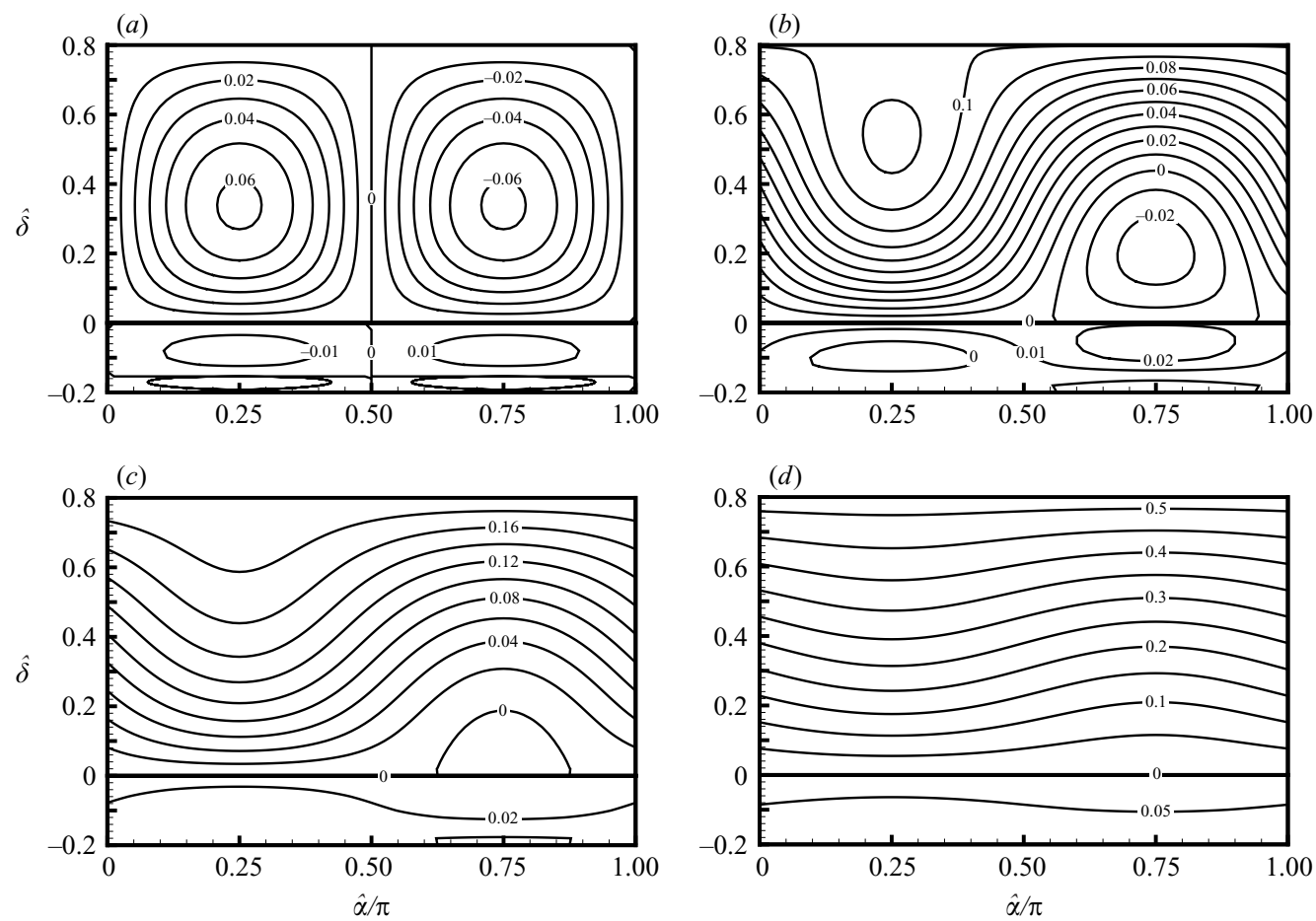

FIGURE 12. Mass transport streamlines in partially standing waves in an open system $(Y=0)$ for $\hat{s}_{m}=0.02$, and (a) $R=1.0,(b) R=0.95,(c) R=0.9$, (d) $R=0.7$.

back downwards to start the next cycle of motion. This kind of alternate up and down motion of the jet can enhance the forward as well as the upward transport of near-bottom substances in the water layer.

We finally show in figure 14 the dependence on $R$ and $\hat{s}_{m}$ of the discharges $\hat{Q}_{w}$ and $\hat{Q}_{m}$ in the two layers when the system is open. As noted earlier in figures 3 and 4 , the lower layer becomes a virtually rigid bed when $\hat{s}_{m}>0.5$. The discharge in the water layer, which therefore has its upper bound at $\hat{s}_{m}=0.5$, decreases with $\hat{s}_{m}$ (i.e. as the lower fluid gets less viscous) for a particular value of $R$. The decrease will, however, become negligible when $\hat{s}_{m}$ drops further from 0.1 . Therefore it can be inferred that the mass transport discharge in the water layer is rather insensitive to the viscosity of the underlying fluid, if the fluid is not too stiff. By contrast, the viscosity of the lower fluid has a more appreciable effect on the discharge of the fluid itself. The discharge in this lower layer may increase considerably by decreasing $\hat{s}_{m}$. Although not shown here, a larger discharge can also result from a smaller density of the fluid. A less concentrated fluid mud, which has a smaller density and viscosity, will therefore tend to drift more readily under surface waves in a water-mud system.

\section{Concluding remarks}

We have presented analytical solutions to the problem of mass transport due to partially reflected long surface waves in a two-layer system, where the domain can be open or closed and the lower fluid is much more viscous than the upper one. The dominant free-surface set-up is the one due to the non-viscous part of motion under 

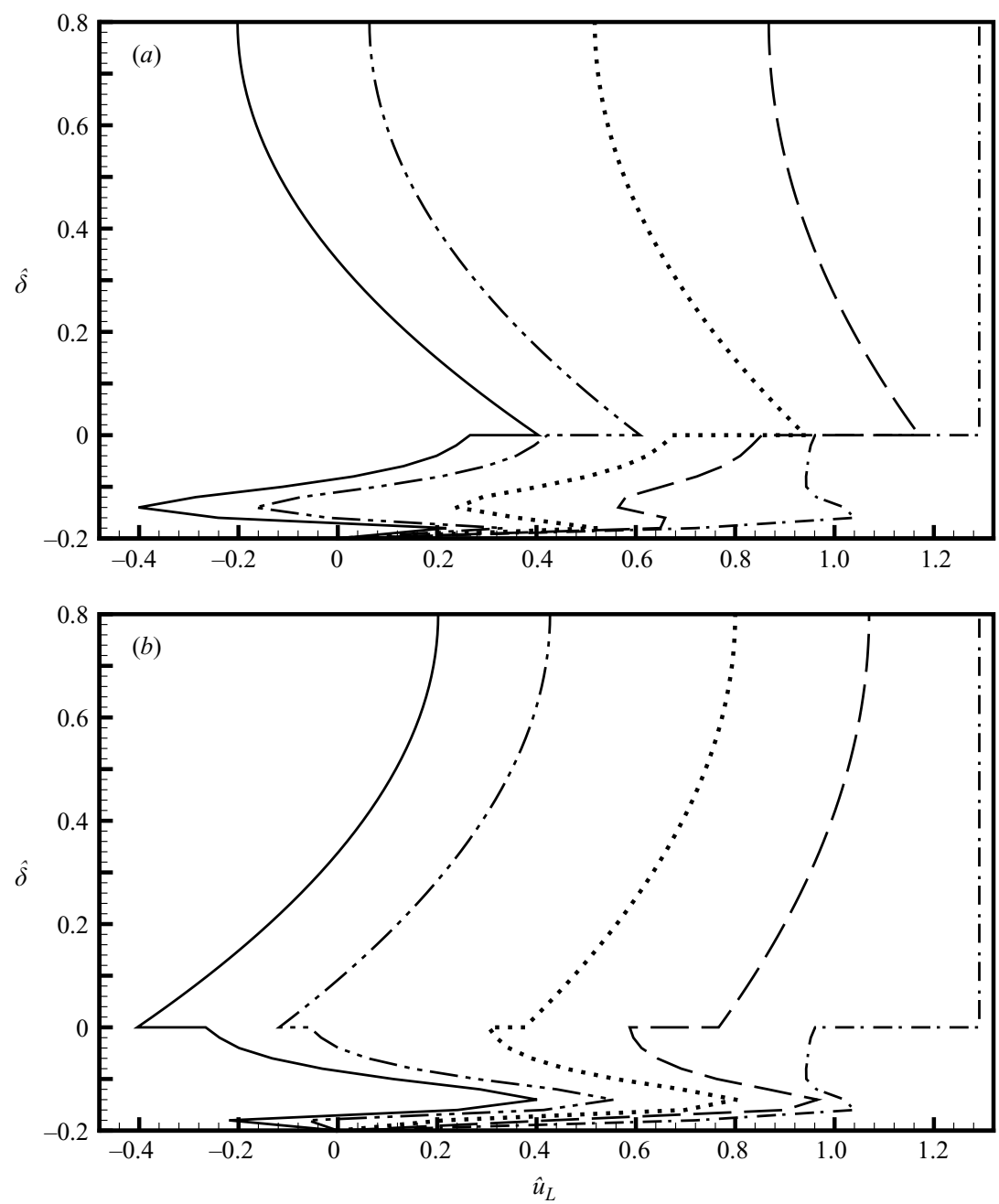

FiguRE 13. Mass transport velocity profiles at $(a) \hat{\alpha}=\pi / 4,(b) \hat{\alpha}=3 \pi / 4$ under reflected waves in an open system $(Y=0)$ for $\hat{s}_{m}=0.02(-, R=1.0 ;-\cdots-R=0.9 ; \cdots, R=0.7 ;-\square$, $R=0.5 ;-\cdot-, R=0.0$ ).

a standing wave. The subdominant set-ups of the free surface and the interface are those induced to produce currents to balance the spatially alternating drifts due to a standing wave and the forward drifts due to a progressive wave when the system is closed. It is remarkable that a Lagrangian set-up will not only have a dramatically different expression from its Eulerian counterpart, but also have a non-zero spatial mean unlike its Eulerian counterpart. It has been shown that the viscosity of the lower fluid can be influential in determining the recirculation structures in a standing wave, and the drift profiles in a progressive wave. In either limiting case of the wave, there exists a particular value of the viscosity of the lower fluid for which the mean motion in the core of the upper layer vanishes completely. Also, in a partially reflected wave, the flow recirculation may persist until reflection is very low when the system is closed, but will rapidly disappear upon departure from perfect reflection when the system is open. In the latter case, when a reflection coefficient is just below unity, 

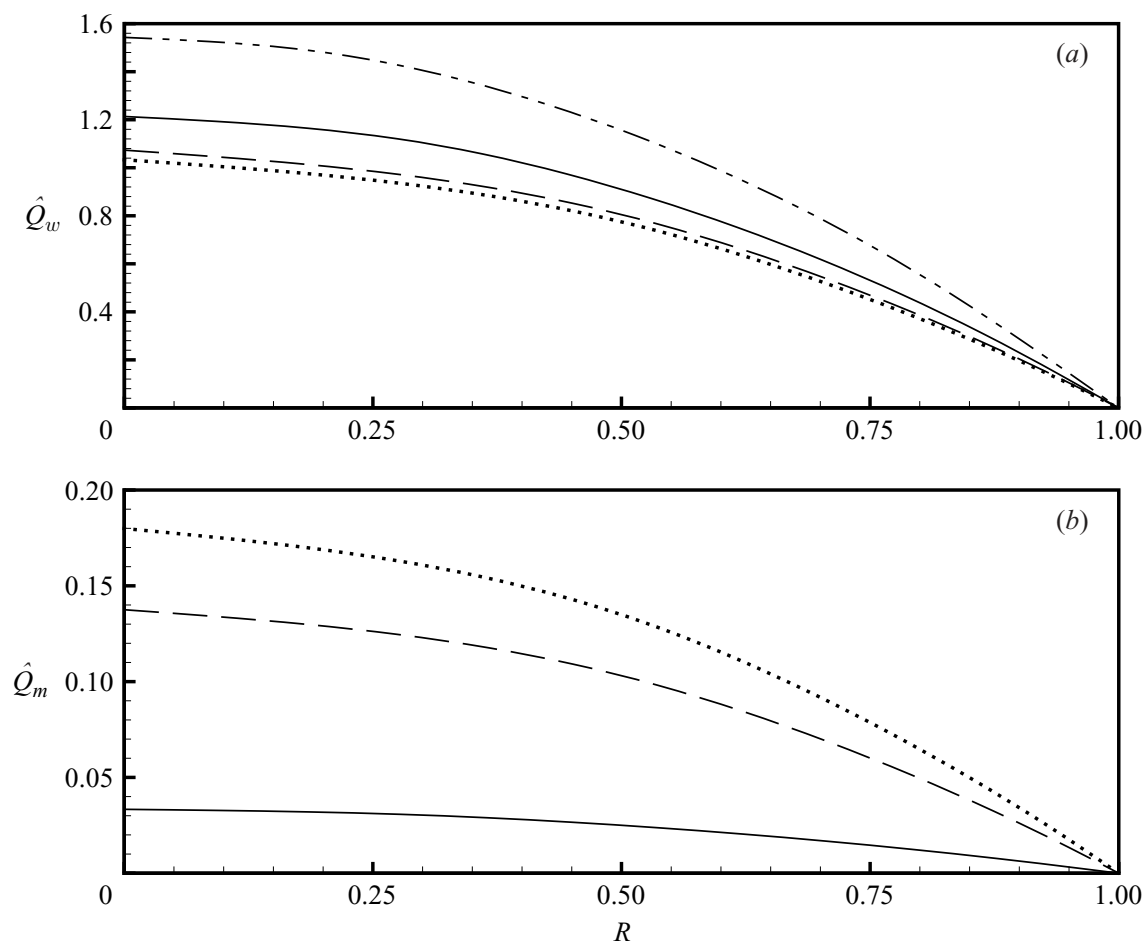

FIGURE 14. Discharges in an open system: (a) upper-layer water $\hat{Q}_{w},(b)$ lower-layer viscous fluid $\hat{Q}_{m}$ as functions of the reflection coefficient $R$ for $\hat{s}_{m}$ equal to $0.5(-\cdots-), 0.2(-), 0.1$ $(--)$, and $0.02(\cdots)$.

a forward jet that shoots alternately up and down can be established in the upper layer.

Extension of the present work to a lower layer of non-Newtonian fluid is worth pursuing. Muds of a sufficiently high solid content are very often shear thinning, or the effective viscosity will decrease with increase in the shear rate. The interfacial setup for the present Newtonian case, as mentioned above, is very tiny in amplitude and of little practical significance in general. By sharp contrast, the response of a shearthinning fluid to surface waves can be dramatically different. Under a sufficiently high load, the wave amplitude and the corresponding mass transport velocity in a layer of shear-thinning fluid can be much larger than when the fluid is Newtonian $(\mathrm{Ng} 2004 b)$. The non-Newtonian rheology will lead to, among others, the formation of a periodic but non-simple-harmonic permanent spatial pattern in the lower layer when under a reflected surface wave (Becker \& Bercovici 2000); this is important to the study of bedform morphology in a coastal environment. The problem is of course nonlinear and the analysis will be more complicated, but interesting and distinct findings are anticipated.

The author would like to acknowledge the useful comments of the three referees. The work by McIntyre (1988) was pointed out to the author by one of them. The present work was supported by the Research Grants Council of the Hong Kong Special Administrative Region, China, through Project Nos. HKU 7081/02E and HKU 7199/03E. 


\section{Appendix}

The functions $f_{D}$ and $f_{A}$ in (4.45) are given by

$$
\begin{aligned}
f_{D}(\delta)= & 2 \operatorname{Im}\left\{\beta_{m} E^{*}\left[G \cosh \beta_{m}\left(\delta+h_{m}\right)+H \sinh \beta_{m}\left(\delta+h_{m}\right)\right]\right\} \\
& +\frac{3}{4}\left|\beta_{m} G\right|^{2}\left\{\cosh 2 \beta_{m r}\left(\delta+h_{m}\right)-\cos 2 \beta_{m r}\left(\delta+h_{m}\right)\right\} \\
& +\frac{3}{4}\left|\beta_{m} H\right|^{2}\left\{\cosh 2 \beta_{m r}\left(\delta+h_{m}\right)+\cos 2 \beta_{m r}\left(\delta+h_{m}\right)\right\} \\
& +\frac{3}{2}\left|\beta_{m}\right|^{2}\left\{\operatorname{Re}\left(H G^{*}\right) \sinh 2 \beta_{m r}\left(\delta+h_{m}\right)-\operatorname{Im}\left(H G^{*}\right) \sin 2 \beta_{m r}\left(\delta+h_{m}\right)\right\}, \\
f_{A}(\delta)= & \gamma A\left[A-\operatorname{Re}\left(\beta_{w} D\right)\right]-|E|^{2}-2 \operatorname{Re}\left\{\beta_{m} E^{*}\left(G \cosh \beta_{m} h_{m}+H \sinh \beta_{m} h_{m}\right)\right\} \\
& +4 \operatorname{Re}\left\{\beta_{m} E^{*}\left[G \cosh \beta_{m}\left(\delta+h_{m}\right)+H \sinh \beta_{m}\left(\delta+h_{m}\right)\right]\right\} \\
& +\frac{3}{2}\left|\beta_{m} G\right|^{2}\left\{\cosh 2 \beta_{m r}\left(\delta+h_{m}\right)+\cos 2 \beta_{m r}\left(\delta+h_{m}\right)\right\} \\
& +\frac{3}{2}\left|\beta_{m} H\right|^{2}\left\{\cosh 2 \beta_{m r}\left(\delta+h_{m}\right)-\cos 2 \beta_{m r}\left(\delta+h_{m}\right)\right\} \\
& +3\left|\beta_{m}\right|^{2}\left\{\operatorname{Re}\left(H G^{*}\right) \sinh 2 \beta_{m r}\left(\delta+h_{m}\right)+\operatorname{Im}\left(H G^{*}\right) \sin 2 \beta_{m r}\left(\delta+h_{m}\right)\right\} .
\end{aligned}
$$

$U_{m D}(\delta)$ and $U_{m A}(\delta)$ in equation (4.46) are given by

$$
\begin{aligned}
U_{m D}(\delta)= & \left(\delta+h_{m}\right)\left[2 \gamma A \operatorname{Im}(d)-\frac{3}{2} \gamma \beta_{w r}|D|^{2}\right] \\
& +2 \operatorname{Im}\left\{\frac{E^{*}}{\beta_{m}}\left[G \cosh \beta_{m}\left(\delta+h_{m}\right)+H \sinh \beta_{m}\left(\delta+h_{m}\right)-G-\beta_{m}\left(\delta+h_{m}\right) \gamma D\right]\right\} \\
& +\frac{3}{8}|G|^{2}\left\{\cosh 2 \beta_{m r}\left(\delta+h_{m}\right)+\cos 2 \beta_{m r}\left(\delta+h_{m}\right)-2-2 \beta_{m r}\left(\delta+h_{m}\right)\left(\sinh 2 \beta_{m r} h_{m}\right.\right. \\
& \left.\left.-\sin 2 \beta_{m r} h_{m}\right)\right\}+\frac{3}{8}|H|^{2}\left\{\cosh 2 \beta_{m r}\left(\delta+h_{m}\right)-\cos 2 \beta_{m r}\left(\delta+h_{m}\right)\right. \\
& \left.-2 \beta_{m r}\left(\delta+h_{m}\right)\left(\sinh 2 \beta_{m r} h_{m}+\sin 2 \beta_{m r} h_{m}\right)\right\} \\
& +\frac{3}{4} \operatorname{Re}\left(H G^{*}\right)\left\{\sinh 2 \beta_{m r}\left(\delta+h_{m}\right)-2 \beta_{m r}\left(\delta+h_{m}\right) \cosh 2 \beta_{m r} h_{m}\right\} \\
& +\frac{3}{4} \operatorname{Im}\left(H G^{*}\right)\left\{\sin 2 \beta_{m r}\left(\delta+h_{m}\right)-2 \beta_{m r}\left(\delta+h_{m}\right) \cos 2 \beta_{m r} h_{m}\right\}, \\
U_{m A}(\delta)= & \left(\delta+h_{m}\right)\left[4 \gamma A \operatorname{Re}(d)-3 \gamma \beta_{w r}|D|^{2}\right] \\
& +\frac{1}{2}\left(\delta^{2}-h_{m}^{2}\right)\left\{|E|^{2}+\operatorname{Re}\left[\left(A-\beta_{w} D\right)\left(\gamma A-2 E^{*}\right)\right]\right\} \\
& +4 \operatorname{Re}\left\{\frac{E^{*}}{\beta_{m}}\left[G \cosh \beta_{m}\left(\delta+h_{m}\right)+H \sinh \beta_{m}\left(\delta+h_{m}\right)-G-\beta_{m}\left(\delta+h_{m}\right) \gamma D\right]\right\} \\
& +\frac{3}{4}|G|^{2}\left\{\cosh 2 \beta_{m r}\left(\delta+h_{m}\right)-\cos 2 \beta_{m r}\left(\delta+h_{m}\right)\right. \\
& \left.-2 \beta_{m r}\left(\delta+h_{m}\right)\left(\sinh 2 \beta_{m r} h_{m}+\sin 2 \beta_{m r} h_{m}\right)\right\} \\
& +\frac{3}{4}|H|^{2}\left\{\cosh 2 \beta_{m r}\left(\delta+h_{m}\right)+\cos 2 \beta_{m r}\left(\delta+h_{m}\right)-2\right. \\
& \left.-2 \beta_{m r}\left(\delta+h_{m}\right)\left(\sinh 2 \beta_{m r} h_{m}-\sin 2 \beta_{m r} h_{m}\right)\right\} \\
& +\frac{3}{2} \operatorname{Re}\left(H G^{*}\right)\left\{\sinh 2 \beta_{m r}\left(\delta+h_{m}\right)-2 \beta_{m r}\left(\delta+h_{m}\right) \cosh 2 \beta_{m r} h_{m}\right\} \\
& -\frac{3}{2} \operatorname{Im}\left(H G^{*}\right)\left\{\sin 2 \beta_{m r}\left(\delta+h_{m}\right)-2 \beta_{m r}\left(\delta+h_{m}\right) \cos 2 \beta_{m r} h_{m}\right\}, \\
& a(\mathrm{~A} 4)
\end{aligned}
$$

and $Q_{m D}$ and $Q_{m A}$ in (4.47) by

$$
\begin{aligned}
Q_{m D}= & \frac{1}{2} h_{m}^{2}\left[2 \gamma A \operatorname{Im}(d)-\frac{3}{2} \gamma \beta_{w r}|D|^{2}\right] \\
& +2 \operatorname{Im}\left\{\frac{E^{*}}{\beta_{m}^{2}}\left[G \sinh \beta_{m} h_{m}+H \cosh \beta_{m} h_{m}-H-\beta_{m} h_{m} G-\frac{1}{2} \beta_{m}^{2} h_{m}^{2} \gamma D\right]\right\} \\
& +\frac{3}{16 \beta_{m r}}|G|^{2}\left\{\sinh 2 \beta_{m r} h_{m}+\sin 2 \beta_{m r} h_{m}-4 \beta_{m r} h_{m}\right. \\
& \left.-2 \beta_{m r}^{2} h_{m}^{2}\left(\sinh 2 \beta_{m r} h_{m}-\sin 2 \beta_{m r} h_{m}\right)\right\}+\frac{3}{16 \beta_{m r}}|H|^{2}\left\{\sinh 2 \beta_{m r} h_{m}\right.
\end{aligned}
$$




$$
\begin{aligned}
& \left.-\sin 2 \beta_{m r} h_{m}-2 \beta_{m r}^{2} h_{m}^{2}\left(\sinh 2 \beta_{m r} h_{m}+\sin 2 \beta_{m r} h_{m}\right)\right\} \\
& +\frac{3}{8 \beta_{m r}} \operatorname{Re}\left(H G^{*}\right)\left\{\cosh 2 \beta_{m r} h_{m}-1-2 \beta_{m r}^{2} h_{m}^{2} \cosh 2 \beta_{m r} h_{m}\right\} \\
& +\frac{3}{8 \beta_{m r}} \operatorname{Im}\left(H G^{*}\right)\left\{-\cos 2 \beta_{m r} h_{m}+1-2 \beta_{m r}^{2} h_{m}^{2} \cos 2 \beta_{m r} h_{m}\right\} . \\
Q_{m A}= & \frac{1}{2} h_{m}^{2}\left[4 \gamma A \operatorname{Re}(d)-3 \gamma \beta_{w r}|D|^{2}\right]-\frac{h_{m}^{3}}{3}\left\{|E|^{2}+\operatorname{Re}\left[\left(A-\beta_{w} D\right)\left(\gamma A-2 E^{*}\right)\right]\right\} \\
& +4 \operatorname{Re}\left\{\frac{E^{*}}{\beta_{m}^{2}}\left[G \sinh \beta_{m} h_{m}+H \cosh \beta_{m} h_{m}-H-\beta_{m} h_{m} G-\frac{1}{2} \beta_{m}^{2} h_{m}^{2} \gamma D\right]\right\} \\
& +\frac{3}{8 \beta_{m r}}|G|^{2}\left\{\sinh 2 \beta_{m r} h_{m}-\sin 2 \beta_{m r} h_{m}-2 \beta_{m r}^{2} h_{m}^{2}\left(\sinh 2 \beta_{m r} h_{m}+\sin 2 \beta_{m r} h_{m}\right)\right\} \\
& +\frac{3}{8 \beta_{m r}}|H|^{2}\left\{\sinh 2 \beta_{m r} h_{m}+\sin 2 \beta_{m r} h_{m}-4 \beta_{m r} h_{m}\right. \\
& \left.-2 \beta_{m r}^{2} h_{m}^{2}\left(\sinh 2 \beta_{m r} h_{m}-\sin 2 \beta_{m r} h_{m}\right)\right\} \\
& +\frac{3}{4 \beta_{m r}} \operatorname{Re}\left(H G^{*}\right)\left\{\cosh 2 \beta_{m r} h_{m}-1-2 \beta_{m r}^{2} h_{m}^{2} \cosh 2 \beta_{m r} h_{m}\right\} \\
& -\frac{3}{4 \beta_{m r}} \operatorname{Im}\left(H G^{*}\right)\left\{-\cos 2 \beta_{m r} h_{m}+1-2 \beta_{m r}^{2} h_{m}^{2} \cos 2 \beta_{m r} h_{m}\right\} .
\end{aligned}
$$

\section{REFERENCES}

Becker, J. M. \& Bercovici, D. 2000 Permanent bedforms in a theoretical model of wave-sea-bed interactions. Nonlinear Proc. Geophys. 7, 31-35.

Crampin, D. J. \& Dore, B. D. 1979 Mass transport induced by standing interfacial waves. Mathematika 26, 224-235.

Dalrymple, R. A. \& LiU, P. L.-F. 1978 Waves over soft muds: a two-layer fluid model. J. Phys. Oceanogr. 8, 1121-1131.

Dore, B. D. 1970 Mass transport in layered fluid systems. J. Fluid Mech. 40, 113-126.

Dore, B. D. 1973 On mass transport induced by interfacial oscillations at a single frequency. Proc. Camb. Phil. Soc. 74, 333-347.

Dore, B. D. $1976 a$ Double boundary layers in standing interfacial waves. J. Fluid Mech. 76, 819-828.

Dore, B. D. $1976 b$ Double boundary layers in standing surface waves. Pure Appl. Geophys. 114, 629-637.

DoRE, B. D. 1978 A double boundary-layer model of mass transport in progressive interfacial waves. J. Engng Maths 12, 289-301.

Iskandarani, M. \& LiU, P. L.-F. 1991 Mass transport in two-dimensional water waves. J. Fluid Mech. 231, 395-415.

Longuet-Higgins, M. S. 1953 Mass transport in water waves. Phil. Trans. R. Soc. Lond. A 245, $535-581$.

Longuet-Higgins, M. S. \& Stewart, R. W. 1964 Radiation stresses in water waves; a physical discussion, with applications. Deep-Sea Res. 11, 529-562.

MCIntyre, M. E. 1988 A note on the divergence effect and the Lagrangian-mean surface elevation in periodic water waves. J. Fluid Mech. 189, 235-242.

MeI, C. C. 1989 The Applied Dynamics of Ocean Surface Waves. World Scientific.

MeI, C. C. \& LiU, K.-F. 1987 A Bingham-plastic model for a muddy seabed under long waves. J. Geophys. Res. 92, 14581-14594.

NG, C.-O. 2004a Mass transport in gravity waves revisited. J. Geophys. Res. 109, C4, C04012, doi:10.1029/2003JC002121.

NG, C.-O. $2004 b$ Mass transport in a layer of power-law fluid forced by periodic surface pressure. Wave Motion 39, 241-259. 
NG, C.-O. \& Fu, S.-C. 2002 On the propagation of a two-dimensional viscous density current under surface waves. Phys. Fluids 14, 970-984.

Piedra-Cueva, I. 1995 Drift velocity of spatially decaying waves in a two-layer viscous system. J. Fluid Mech. 299, 217-239.

Pierson, W. J. 1962 Perturbation analysis of the Navier-Stokes equations in Lagrangian form with selected linear solutions. J. Geophys. Res. 67, 3151-3160.

SakakiYama, T. \& Bijker, E. W. 1989 Mass transport velocity in mud layer due to progressive waves. J. Waterway Port, Coastal Ocean Engng 115, 614-633.

ÜnLÜATA, Ü. \& MeI, C. C. 1970 Mass transport in water waves. J. Geophys. Res. 75, 7611-7618.

WeN, J. \& LiU, P. L.-F. 1995 Mass transport of interfacial waves in a two-layer fluid system. J. Fluid Mech. 297, 231-254. 